\title{
Travail de l'anthropologue et travail des témoins, Moskitia 1982-2007
}

The work of the anthropologist and the work of witnesses, Moskitia 1982-2007

\section{Gilles Bataillon}

\section{(2) OpenEdition}

12 Journals

\section{Édition électronique}

URL : http://journals.openedition.org/conflits/17448

DOI : $10.4000 /$ conflits. 17448

ISSN : $1777-5345$

Éditeur :

CCLS - Centre d'études sur les conflits lilberté et sécurité, L'Harmattan

\section{Édition imprimée}

Date de publication : 1 septembre 2009

Pagination : 129-166

ISBN : 978-2-296-09110-8

ISSN : 1157-996X

\section{Référence électronique}

Gilles Bataillon, «Travail de l'anthropologue et travail des témoins, Moskitia 1982-2007 », Cultures \& Conflits [En ligne], 74 | été 2009, mis en ligne le 28 octobre 2010, consulté le 30 mars 2021. URL

http://journals.openedition.org/conflits/17448; DOI : https://doi.org/10.4000/conflits.17448 


\section{Travail de I'anthropologue et travail des témoins, Moskitia 1982-2007}

\section{Gilles BATAILLON}

Gilles Bataillon est sociologue à l'Ecole des hautes études en sciences sociales (EHESS-Paris) et an Centro de Investigacion y Docencia Economica (CIDEMexico).

Comment décrire et comprendre une guerre civile, comme celle que connut la Moskitia nicaraguayenne de 1981 à 1989, quand il n'en existe pas d'archives accessibles et que les principaux acteurs du conflit sont toujours des personnages politiques de premier plan ? Comment interroger les témoins et les acteurs ? Que sont-ils prêts à raconter et à quelles règles obéissent leurs récits ? Comment utiliser ces récits et que révèlent-ils tant sur le passé que sur le présent ? Autant de questions à l'origine de ces réflexions sur le travail de terrain que j’ai mené en Moskitia de 1982 à 2007 1. Présent dans les maquis miskitus en 1984, j'ai commencé par m'interroger dès cette époque sur les limites de ce que pouvaient percevoir les acteurs comme les observateurs lorsqu'ils étaient immergés dans l'événement ${ }^{2}$. Les conditions de guerre favorisaient un certain type de discours qui faisait peu de place à l'introspection et à la réflexion individuelle. La parole n'était pas simplement censurée pour des raisons de prudence évidente, mais plus fondamentalement, le je et la libre réflexion du locuteur étaient déniés par l'incorporation des combattants dans un corps, la guérilla. L'observateur lui-même peinait à échapper à cette emprise.

1. On trouvera un complément d'information sur la façon dont s'est déroulé cette enquête dans : Bataillon G., Enquêtes sur une guérilla (Nicaragua 1982-2007), Paris, Le Félin, 2009, et un panorama du contexte dans lequel a surgi la Contra (l'opposition armée aux sandinistes) dans un article publié dans un numéro antérieur de Cultures E Conflits : Bataillon G., « Réflexions sur l'action armée et la constitution d'acteurs-politico-militaires : contras et recontras nicaraguayens 1982-1993 », Cultures E Conflits, n¹2, 1993-1994, pp. 63-103.

2. Bataillon G., « Moskitia 1984 », Volonta, n³, 1985, Milan. 
C'est dans un contexte marqué par une soif d'interrogations neuves, et par des aspirations à des réformes politiques et sociales nées pendant les années de guerre, que j'effectuai toute une série de séjours en Moskitia à partir de l'hiver 1997-1998. Venant tout d'abord de six mois en six mois, puis seulement une fois par an à partir de 2001, je fis une douzaine de séjours de 1998 à 2007. Mes enquêtes débutèrent à Puerto Cabezas et dans les communautés voisines de cette petite ville, pour se poursuivre sur le Rio Coco, tantôt en compagnie d'anciens guérilleros comme Mono, tantôt seul. Multipliant les conversations avec les personnes les plus diverses, je recueillis une bonne centaine d'" autobiographies modèles ", au premier chef auprès d'anciens guérilleros et de membres de leurs familles, mais aussi auprès de Miskitus et de métis hispanophones proches des sandinistes. Je décidai parallèlement de commencer à présenter les résultats de mes recherches aux habitants de la Moskitia, tout d'abord en faisant une conférence, puis à travers une série de cours dans l'une des deux petites universités de Puerto Cabezas et, ensuite, en faisant circuler et en discutant les premiers matériaux que je publiai à la fois en français et en espagnol. Je réalisai enfin deux enquêtes en collaboration avec d'anciens guérilleros miskitus pour le compte d'institutions internationales, l'une en collaboration avec Mono pour le compte de l'Organisation des Etats américains (OEA) en août 2000, l'autre en collaboration avec plusieurs anciens guérilleros pour la Banque mondiale en 2002. Ce travail de construction de récits de vie avec les acteurs d'un conflit armé ne m'a pas seulement permis de restituer la part de l'aléatoire et de l'accidentel, comme le poids du contexte dans les processus qui amènent des individus à s'engager dans une guerre civile. Il m'a aussi conduit à m'interroger sur la façon dont le contexte social pèse sur la réflexion des acteurs et sur les modalités du récit du passé qu'ils sont prêts à construire, comme sur leurs appréciations du présent. J’ai ainsi repéré la façon dont tout le travail de remémoration de la guerre ou d'autres événements, auquel j'invitai mes interlocuteurs, s'appuyait sur la tradition introspective du piétisme morave, religion à laquelle se sont convertis les Miskitus depuis le début du $\mathrm{XX}^{\mathrm{e}}$ siècle $^{3}$. Je montre aussi comment, dans leurs manières même de

3. On verra sur cette conversion les ouvrages suivants : Schneider H.G., La Mosquitia, historia de la Unitas fratrum en La Mosquitia (1849-1898), Managua, CIETTS, [edición original en alemán, Herrnhut, 1899], 1998) ; Mueller K., Among Creoles, Miskitos and Sumos. Eastern Nicaragua and its Moravian Missions, Bethlehem, The Comenius Press, 1932; Wilson J., Obra morava en Nicaragua: trasfondo y breve historia, Managua, Unión, 1990 ; et l'article Bataillon G., «De Sandino aux contras. Formes et pratiques de la guerre au Nicaragua », Annales, maijuin 2005, pp. 653-688. Sur l'histoire des Miskitus, les travaux fondamentaux restent ceux de Conzemius : Conzemius E., Ethnographical Survey of the Miskito and Sumu Indians of Honduras and Nicaragua, Washington, Bureau of American Ethnology, bulletin n ${ }^{\circ} 106,1932$. Les travaux de Helms restent également très importants : Helms M., "Of Kings and Contexts: Ethnohistorical Interpretations of Miskito Political Structure and Function", American Ethnologist, vol. 13, n³, 1986, pp. 506-523 ; "Miskito Slaving and Culture Contact: Ethnicity and Opportunity in an Expanding Population", Journal of Anthropological Research, vol. 39, 1983, pp. 179-197 ; Asang: adaptaciones al contacto cultural en una sociedad misquito, México, Instituto Indigenista Interamericano, 1976 ; Asang: Adaptations to Culture Contact in a Miskito Community, Gainsville (Florida), University of Florida Press, 1971 ; "The Cultural Ecology of a Colonial Tribe”, Ethnology, vol. VIII, n¹, 1969, pp. 76-84. Citons aussi les travaux de Nietschmann : Nietschmann B., Between Land and Water: The Subsistence Ecology of 
raconter, comme dans leurs soucis d'une histoire plurielle, les Miskitus reprennent à leur compte certaines traditions pluralistes du protestantisme. Ces pages offrent ainsi une réflexion non seulement sur l'histoire des Miskitus au $\mathrm{XX}^{\mathrm{e}}$ siècle, mais sur le travail d'enquête sur une guerre civile et sur la manière dont la mise en récit du passé peut s'effectuer.

\section{A la recherche d'« autobiographies modèles "}

Mon séjour s’est ouvert par les fêtes de Noël de 1997 et du Nouvel An 1998 à Puerto Cabezas, et j’arrivai très décidé à recueillir avant tout les « autobiographies modèles » des gens dont j'avais arrêté la liste quelques mois plus tôt avec Samuel Kittlé et Adam Artola, deux des commandants avec lesquels je m’étais lié d'amitié lors de mes premiers séjours dans la guérilla en 1984. J'entendais également poursuivre le travail, déjà bien entamé, avec eux. L'ambiance de Puerto, le contexte des fêtes de fin d'année et les desiderata de mes interlocuteurs m'obligèrent très vite à modifier mon projet d'enquête. Ayant fait savoir à Samuel et Adam que j'arriverais à la mi-décembre, tous deux eurent à cœur d'annoncer mon arrivée sur les ondes de Radio Miskut. Ce faisant, ils contribuèrent véritablement à redessiner les contours de mon projet. Ils évoquaient très chaleureusement mon projet d'enquête, rappelaient mes liens avec les partisans de la Reforma ${ }^{4}$, mes cours d'histoire des guerres de guérilla auprès des cadres de la guérilla en juillet 1984, et non seulement ils firent de moi, que je le veuille ou non, un interprète autorisé de l'histoire des Miskitus, mais ils apportèrent de sérieuses retouches au personnage de l'observateur scientifique étranger que je prétendais camper. Je fus immédiatement réinscrit dans le paysage politique du passé comme du présent. "Allié objectif » des gens de la Reforma à l'époque de mes cours, on m'associa dès mon arrivée aux individus gravitant autour de radio Miskut. Je n'étais plus uniquement un professeur étranger revenant poursuivre une enquête qui était presque impossible une vingtaine d'année auparavant : cette enquête fut présentée à ceux que je vins questionner comme le prolongement de ma présence

the Miskito Indians, Eastern Nicaragua, Nueva York / Londres, Seminar Press, 1973 ; The unknown war: The Miskito Nation, Nicaragua and the United States, Boston, University Press of America, 1989. Enfin, nous pouvons ajouter: Hale C.R., Resistance and Contradiction: Miskitu Indians and the Nicaraguan State, 1894-1987, Stanford, Stanford University Press, 1994 et Baron L.P., Shipwrecked identities. Navigating Race on Nicaragua's Mosquito Coast, Rutgers University Press, 2006.

4. Le mouvement d'opposition aux deux leaders miskitus, Steadman Fagoth et Brooklyn Rivera, fondateurs en 1979 de l'organisation indianiste Miskito Sumu Rama Sandinista asla Takankal Miskito Sumu Rama Sandinista tous unis (MISURASATA), devenus par la suite les dirigeants des deux guérillas mikitues rivales. Celle dirigée par Fagoth prit le nom de MISURA pour marquer de la façon la plus nette possible son opposition sans concession aux sandinistes, tandis que l'autre, dirigée par Rivera, conserva le nom de MISURASATA, pour au contraire marquer sa volonté de ne pas complètement rompre avec les sandinistes et laisser la porte ouverte à des négociations. Voir Bataillon G., "Comandantes, état-major et guérilleros : jeux du pouvoir à l'intérieur de la guérilla miskitu (Nicaragua 1981-1984) ", Cabiers des Amériques latines, n³6, 2001, pp. 127-159 et Bataillon G., " Guerre et assemblée, découverte et apprentissage de la démocratie chez les Miskitus nicaraguayens (1981-1988) ", Cultures E Conflits, $\mathrm{n}^{\circ} 47,2002$, pp. 195-215. 
professorale aux cotés des guérilleros en 1984 et du travail réflexif que nous y avions accompli. Mon séjour s'inscrivit donc dans une double démarche : celle de la construction d'une histoire et d'un bilan de la guerre contre les sandinistes et des mobilisations antérieures, mais aussi celle d'une possible réflexion sur le présent, voire d'un appui dans des luttes politiques du moment. Du coup, plusieurs personnes que je voulus interviewer n'entendirent nullement borner la conversation à l'établissement de leur récit de vie ; ils souhaitèrent au moins autant poursuivre des conversations qui furent de véritables dialogues. Eux aussi voulurent pouvoir formuler des questions, tant sur mon projet intellectuel (pourquoi voulais-je écrire sur leur histoire ? Qu'allais-je écrire ?) que sur mes appréciations à propos de la situation présente. A l'inverse, certains proches de Steadman Fagoth et de Brooklyn Rivera et bon nombre de sandinistes plus encore me considérèrent au départ avec une certaine suspicion quand j'exprimai le souhait de m'entretenir avec eux.

Malgré ces quelques réserves, et à la différence des expériences que j’avais pu faire précédemment au cours desquelles les paroles avaient souvent été chichement mesurées, ce fut tout le contraire qui se produisit. Non seulement mes anciennes connaissances vinrent me parler, mais nombre de parfaits inconnus le firent à leur tour très volontiers, que ce soit à l'instigation de Mono et de Wari ou d'autres amis des années 1980. Je fus donc, comme jamais auparavant, face à un trop-plein d'informations et d'offres de conversations. Je dus plus d'une fois recevoir trop vite des personnes envoyées par des tiers, leur donner rendez-vous pour les jours suivants, voire les derniers jours de mon séjour, ou leur promettre de m'entretenir avec eux lors d'un prochain voyage.

Poursuivant mon élaboration d' " autobiographies modèles » avec Samuel et Adam, je me lançai dans le même travail narratif avec d'autres anciens guérilleros. Je rencontrai ainsi plus d'une dizaine d'entre eux : Santiago/Coyote, l'archiviste de la guérilla, dont la chronologie constituait pour moi une manière de faire le croquis de l'histoire de celle-ci; Benigno/Canceroso, qui avait été mon traducteur pour les Mayangnas à El Atillo ${ }^{5}$; Tata, le responsable de Radio Miskut, déjà à la tête de celle-ci durant la guerre ; Blas, le commandant en chef élu démocratiquement à Rus Rus, fort occupé à suivre des études pour ouvrir son cabinet d'avocat; Minor Jimenez, un second de Mono devenu depuis comptable; Felix Suarez, qui avait été un des rares Miskitus à avoir reçu un entraînement de commando parachutiste à l'époque où les Américains avaient fait miroiter aux contras la possibilité de réitérer une opération comme celle qu'ils avaient réalisée à la Grenade en 1983; Alvaro Cirilio Wilson, lui aussi un ancien d'El Atillo, pasteur à la vocation très prosaïque ; Julian Smith, un des rares à avoir hissé les couleurs rouge et noire du FSLN

5. La guérilla comptait non seulement des cadres miskitus, mais également des cadres de l'ethnie voisine des Mikitus, les Mayas, dont certains ne parlaient pas, ou mal, l'espagnol et conversaient en miskitu avec le restant des guérilleros. 
dans sa communauté le jour de la chute de Somoza, devenu par la suite un activiste de MISURASATA et parmi les premiers à partir en exil, vivant à l'époque du métier de taxi, tout en étant l'un des membres les plus éminents du conseil des anciens du temple morave le plus important de Puerto; mes amies ex-guérilleras ; les sœurs Bob, Chica et Silvia, toutes deux mères divorcées vivant d'emplois informels, devenues les têtes de file d'une fratrie féminine installée dans la maison de leurs parents à Puerto ; et enfin Suzy, elle aussi divorcée et élevant seule ses enfants grâce à son salaire d'infirmière. Plusieurs me présentèrent des parents plus âgés, dont ils souhaitèrent que je recueille les témoignages. L'oncle de Chica et Silvia, Rogelio Bob, me raconta ainsi en miskitu, tandis que Chica traduisait, ce qu'avait été sa jeunesse à Asang à l'époque des guerres de Sandino et me traça des portraits peu flatteurs de ses hommes. $\mathrm{Si}$, me dit-il, ce dernier avait été "un hombre decente ", ses subordonnés avaient pour beaucoup laissé le souvenir de « voleurs » faisant non seulement main basse sur leurs quelques richesses, mais abusant volontiers des jeunes filles et réquisitionnant les hommes comme piroguiers, ou comme porteurs.

J'interviewai aussi très longuement plusieurs personnages clés de l'histoire de la guerre, Tillith Mollins et son épouse Laurita, Kenneth et Nicodemo Serapio, ainsi que les membres du conseil des anciens et certains de leurs jeunes assesseurs. Je découvris ainsi la manière dont le révérend Tillith Mollins et sa femme Laurita, une des premières infirmières nicaraguayennes, avaient été des figures de proues de l'Eglise morave sur le Rio Coco, dans la région de Raïti et de Waspam. Le révérend Tillith avait été en outre l'agent d'un véritable réveil miskitu. Il s'était mobilisé sans compter au sein de coopératives agricoles dans les années 1960 et avait été à l'initiative du premier mouvement indianiste, l'Alliance pour le progrès miskitu et sumu (ALPROMISU), dont il avait été un des fondateurs en 1973. Il avait aussi été fort actif lors des mobilisations organisées à Waspam et à Puerto Cabezas pour exiger la libération des dirigeants et des militants de MISURASATA emprisonnés en février 1981, puis il avait pris la fuite au Honduras au lendemain des premières échauffourées avec les sandinistes à la clôture de la campagne d'alphabétisation en langue vernaculaire. Il était enfin devenu une sorte de messie au sein de la guérilla. Kenneth Serapio, un médecin miskitu né à Raïti, responsable du petit hôpital de campagne, et depuis réinstallé à Puerto, m'introduisit à l'histoire de son village, où avaient eu lieu les premiers combats entre la guérilla et les sandinistes. Non content de me donner ses impressions sur leurs années de lutte, il me brossa un tableau plein de justesse et de nuances de la difficulté de la société costena à renouer avec une vie normale au lendemain de dix années de guerre. Il m'expliqua en outre comment et pourquoi il s'était lancé en politique en fondant un parti, le Parti pour l'unité de la Côte atlantique (PAMUC), cherchant à promouvoir le développement local sur la base de l'union entre les guérilleros de la Reforma et les sandinistes dissidents. Il m'invita enfin à collaborer avec l'université qui venait de se créer à Puerto Cabezas, 
la Bluefields Indian E Caribbean University (BICU), dont il avait été élu recteur. Je pris en note les dires du responsable des premières opérations de guerre contre les sandinistes à Raïti, Nicodemo Serapio. Originaire de ce village, parent très éloigné de Kenneth, cet ancien garde national de Somoza s'était très rapidement imposé aux jeunes apprentis guérilleros et s'était presque aussi rapidement heurté avec Fagoth et les autres gardes nationaux qui l'entouraient. En accord avec Tigre, il avait saisi la première occasion pour faire sécession et constituer des maquis autonomes, les Astros/Astres et les Cruces/Croix. Dès lors, fort de ses connaissances militaires, comme de ses dons de voyance, il avait lancé les premiers coups de main de la guérilla contre les sandinistes; coups de mains auxquels l'armée avait répliqué par la tuerie de Leimus (23-24 décembre 1981) ${ }^{6}$, puis par la destruction des villages du Rio Coco et le déplacement forcé des populations lors des premières semaines de l’année 1982.

Ce premier panorama des mobilisations des jeunes Miskitus à l'ombre de l'Eglise morave dès les années 1970 me permit de brosser un tableau assez complet des premiers moments de la révolution sandiniste en Moskitia, notamment de la fuite de la garde nationale, de l'arrivée des sandinistes, du mélange d'expectatives surgies du renversement de Somoza, et des craintes des plus vieux face à ce qu'ils percevaient comme un possible retour des «mauvais lieutenants du vieux Sandino ». Je saisis les jeux forts complexes de rivalités mimétiques entre, d'une part, les responsables et les activistes du nouveau mouvement indianiste MISURASATA et, d'autre part, les autorités locales sandinistes toutes hispanophones et venues de la partie pacifique du pays. Je me rendis aussi compte à quel point, tout au long de la guerre, un très grand nombre de mes interlocuteurs avaient été pris dans des désirs contradictoires. Je constatai aussi combien certaines de leurs actions n'étaient compréhensibles que si elles étaient inscrites dans ces contradictions. Enfin, je confirmai bon nombre des impressions et des observations sur le style de vie des guérilleros et sur leurs visées, faites lors de mes séjours précédents dans la guérilla (1984 et 1985).

A m'entretenir avec les membres du Conseil des anciens et certains de leurs jeunes assesseurs, je pris conscience de la subtilité des jeux de pouvoir au lendemain de la guerre et de tout un travail de redéfinition de l'identité miskitue. Affirmant assez justement que, face à certains errements des élus des conseils régionaux et des conseils municipaux, le Conseil restait une instance de contre-pouvoir et de forum critique, les anciens et leurs assesseurs me firent parallèlement une grande leçon d'histoire sur le passé Miskitu et, plus généralement, sur celui de la Côte et du Nicaragua. Je les entendis ainsi évo-

6. Voir Bataillon G., «Le Nicaragua et les Indiens Miskitos », Esprit, Paris, ${ }^{\circ} 7 / 8$, juillet-août 1982, pp.145-153; « Le Nicaragua et les Indiens de la Côte atlantique », Esprit, Paris, n 7 , juillet 1983, pp.146-161 ; "Astros et cruces ", in Bataillon G., Merklen D. (dirs), Expériences limites, ruptures et mémoires; dialogues avec l'Amérique Latine, Paris, Karthala (sous presse). 
quer des "souvenirs immémoriaux » venus tous droits de lectures hâtives et tronquées des travaux de German Romero ${ }^{7}$ ou de la revue Wani ${ }^{8}$. Ainsi me présentèrent-ils des visions passablement discutables de ce qu'avait été le territoire miskitu, historiquement. Arguant de la présence de toponymes miskitus sur la rive orientale du grand lac du Nicaragua, ils redessinèrent une sorte de grande Moskitia englobant tous les territoires occupés à des époques fort différentes par les ancêtres des miskitus, faisant de la sorte bon marché de la présence d'autres groupes amérindiens souvent pris en tenailles entre les entreprises colonisatrices espagnoles et les raids des Miskitus entre le XVIe et le $\mathrm{XIX}^{\mathrm{e}}$ siècles. De même, s’ils furent très diserts sur la " démocratie indienne » dont le Conseil des anciens était, à les croire, l'incarnation, depuis des «temps immémoriaux » là encore, ils restèrent muets sur la participation des Miskitus aux trafics d'esclaves à l'époque coloniale.

Je multipliai parallèlement les conversations informelles, à la fois aux marges de mes entretiens, notamment en les préparant avec mes futurs interlocuteurs, à leur issue, et enfin lors des fêtes de fin d'année que je passai avec Adam, sa famille et d'autres anciens guérilleros. Ces conversations, durant lesquelles je ne pris jamais de note, et dont je m'efforçai par la suite de restituer la teneur dans mon journal de bord, me plongèrent immédiatement au cœur des changements sociopolitiques dont les Miskitus étaient parties prenantes, parfois à leur corps défendant, parfois de fort bonne grâce, depuis la fin de la guerre. Dans les propos de mes interlocuteurs, l'heure était incontestablement à une sorte de démocratisation de la société, Miskitus et Mayangnas pouvant désormais accéder à des places et emplois qui leur étaient autrefois interdits. Le fait était d'autant plus notable que le nombre de postes de responsabilité politique, souvent fort bien rémunérés, s'étaient multipliés du fait de la création des conseils régionaux et de la mise en place de conseils municipaux élus. Le départ des commerçants chinois dès la chute de Somoza avait là aussi créé des opportunités pour un certain nombre de Miskitus. La mise en place d'une éducation bilingue avait ouvert des possibilités nouvelles pour des carrières d'instituteurs. Enfin, la mise à l'honneur du multiculturalisme, la volonté des agences internationales de coopération de contribuer à la reconstruction de la côte atlantique et de promouvoir des opérations de développement local, avaient permis la floraison de toute une série d'ONG. Celles-ci représentaient une source d'emplois très importante, souvent assez bien rémunérés, pour des travailleurs sociaux et du personnel de service. Différentes personnes, passées par des études secondaires, autrefois instituteurs pour certaines, responsables politiques ou militaires, liées au monde sandiniste comme à celui de la Contra, constituaient de fait une couche de nouveaux petits notables en rivalité avec le

7. Romero Vargas G., Historia de la Costa Atlántica, Managua, CIDCAUCA, 1996 ; Las sociedades del Atlántico de Nicaragua en los siglos XVII y XVIII, Managua, Colección Cultural, Banco Nicaragüense, 1995.

8. Publiée par le Centro de Investigacion y Documentacion de la Costa Atlantica (CIDCA) depuis fin décembre 1984, cette revue est aujourd'hui publiée en association par le CIDCA et la UCA. 
monde plus traditionnel des commerçants. Ces nouvelles fonctions d'instituteurs ou de travailleurs sociaux étaient avant tout conçues par beaucoup comme un tremplin pour entrer en politique. Ces nouvelles opportunités, liées à l'arrivée de ressources financières émanant du gouvernement central, comme de la coopération internationale, alliées à des habitudes de prévarication nées pendant la guerre, avaient malheureusement aussi permis le développement d'une corruption tous azimuts. Celle-ci faisait d'ailleurs bon ménage avec le commerce illégal des bois précieux et celui de la cocaïne, où étaient impliqués de très hauts responsables politiques et policiers, sandinistes et excontras. Du coup, la plus grande partie des élus locaux considéraient leurs postes comme autant de sinécures et, pensant que ces multiples bonanzas ne dureraient qu'un temps, ils mettaient donc les bouchées doubles pour piller les ressources affectées à la région, ou prélever de très importantes dîmes sur les activités illégales 9 . Ce faisant, comme me le firent remarquer Mono et certains de mes interlocuteurs, toute une éthique du travail, de l'effort et du contrôle de soi, au cœur de l'enseignement de l'Eglise morave, était battue en brèche au profit de la mise à l'honneur d'une sorte de cynisme au jour le jour. Et, comme nous le commentâmes très en détail, il n'y avait pas là simplement une perte des valeurs morales, mais bien plus : l'affaissement d'une foi en l'avenir et la mise à mal des idéaux qui avaient été les leurs au moment de la Reforma. Ils relièrent d'ailleurs souvent ces comportements à ce qu'ils nommaient «les traumas de la guerre». Pour eux, ces comportements prévaricateurs étaient en effet apparus durant la guerre et s'étaient développés du fait d'un sentiment de très grande précarité. Ils tracèrent d'ailleurs des parallèles entre le sort des populations déplacées dans les hameaux stratégiques et celui des réfugiés. Les uns et les autres avaient fait l'expérience de façon répétée et constante d'un mélange de promesses et d'engagements non tenus de la part des autorités civiles et militaires, sandinistes et contras, ainsi celle de la violence la plus nue lors de l'incendie de leurs villages par l'armée sandiniste, puis de la perte de tous leurs repères ordinaires, à la fois pour ceux qui étaient arrivés dans les camps de regroupements sandinistes, et pour ceux fuyant au Honduras. Tous, enfin, avaient pris l'habitude de vivre dans un assistanat, plus ou moins chiche, qui avait largement contribué à développer une irresponsabilité chronique chez tous ceux qui n'avaient jamais été socialisés dans cette éthique du travail, propre aux Miskitus depuis leur conversion au protestantisme. D'autres, tout en partageant ces constats, avaient manifestement décidé de jouer une sorte d'adaptation cynique à ce nouveau contexte. Ils me dirent ainsi très franchement qu'il fallait être "fort bête et très irréaliste " pour ne pas accepter de faire de "bonnes affaires " quand les possibilités se présentaient. Ils ajoutèrent que "si eux n'en profitaient pas, ce seraient d'autres ", et qu'en conséquence "des comportements moraux n'avaient plus aucun sens, car de toute façon l'heure était aux arrangements » 10. Par-delà leurs différences, les

9. Voir Frühling P., Gonzalez M., Buvollen P., Etnicidad y Nación. El desarollo de la autonomía de la Costa Atlantica de Nicaragua, F\&G Editores, Guatemala, 2007. 
raisonneurs cyniques et ceux qui étaient alors soucieux de réhabiliter l'éthique du travail et de la responsabilité me parurent partager un même intérêt pour la chose politique. Le jeu électoral, la nécessité d'apprendre à cohabiter avec les sandinistes, que beaucoup avaient cru pouvoir chasser par les armes...; tout cela fournissait la matière à d'innombrables conversations et à des actions fort prosaïques pour les uns, plus politiques pour les autres. Je découvris la façon dont les thématiques et les préoccupations, qui avaient été au centre de mes discussions avec Mono et les autres futurs artisans de la Reforma, resurgissaient dans les débats concernant le jeu politique, local ou national, et dans ceux touchant au futur économique de la Côte atlantique. J'assistai également à l'essor de deux phénomènes appelés à prendre une extension considérable, qui là aussi faisaient l'objet de multiples conversations : l'urbanisation de la société miskitue et la multiplication des migrations temporaires vers le Costa Rica, la Caraïbe ou les Etats-Unis et le Canada. Jusqu'à la révolution sandiniste de 1979, les Miskitus avaient été avant tout des ruraux : ils n'étaient qu'un nombre réduit à Puerto Cabezas, où les Créoles étaient les plus nombreux, et à Waspam, le siège de nombreux commerces aux mains des Chinois. Managua comptait certes quelques familles miskitues, mais fort peu nombreuses ${ }^{11}$. Au lendemain de la guerre, beaucoup de Miskitus avaient préféré se réinstaller en ville, à Puerto Cabezas où la population avait décuplé, ou à Waspam où la population avait aussi considérablement augmenté. De même, les Miskitus partis à Managua durant les années de guerre y étaient, pour beaucoup, non seulement restés, mais avaient été rejoints par des parents. $\mathrm{Si}$, avant les années 1980, les Miskitus avaient eu l'habitude des migrations temporaires vers la zone des mines, vers la partie pacifique du pays, et si certains s'étaient embarqués comme marins, le fait que, dorénavant, de très nombreuses familles comptaient sur les envois d'argent d'un proche parti à l'étranger constituait sans conteste une nouveauté qui bouleversait bien des habitudes. Je découvris parallèlement comment les Miskitus faisaient l'expérience d'un renouveau religieux, lequel mêlait des phénomènes extraordinairement divers en apparence. Les années de guerre avaient manifestement ouvert le champ à un regain de pratiques shamaniques, jusqu'alors soigneusement tenues à l'écart par l'Eglise morave. Ces pratiques, loin de disparaître avec la fin des combats, étaient devenues autant de manières ordinaires de combattre l'infortune et le malheur, d'autant que les églises pentecôtistes pratiquaient des rituels de cure collective qui leur offraient une place certaine. Par ailleurs, ces

10. Bataillon G., «Cambios culturales y sociopoliticos en las comunidades Mayangnas y Miskitus del RioBocay y el alto Rio Coco ", Journal de la société des américanistes, n ${ }^{\circ} 87$, 2001, Paris, pp. 376-392 ; "Wangki/Rio Coco : de l'après-guerre aux catastrophes naturelles ", Journal de la société des américanistes, $\mathrm{n}^{\circ} 88,2002$, Paris, pp. 260-278.

11. Sur les questions démographiques, on se référera à Hale C.R., Gordon E.T., "Costeño Demography: Historical and Contemporary Demography of Nicaragua's Atlantic Coast", in CIDCA (ed.), Ethnic Groups and the Nation State: The Case of the Atlantic Coast in Nicaragua, Stockholm, Université de Stockholm, 1987, pp. 7-31. Sur l'histoire de la Côte atlantique, on se réfèrera outre aux travaux déjà cités de G. Romero Vargas, de M. Helms, de C.R. Hale, Charles R., et de P.L. Baron. 
églises peu ou pas présentes avant les années 1980, étaient devenues de rudes concurrentes de l'Eglise morave et ralliaient chaque jour de nouveaux fidèles. Du coup, les débats théologiques se multipliaient en son sein, et certains dogmes et pratiques autrefois indiscutables faisaient désormais l'objet de commentaires passionnés.

\section{Questions et remises en causes}

Le dépouillement et la mise en ordre de mes premiers témoignages et observations et un nouveau séjour sur le terrain en novembre 1998 me permirent de commencer un travail d'analyse comparative de ces différents matériaux. Si mon projet initial était de constituer une série d' "autobiographies modèles » des anciens guérilleros miskitus, je ne tardai pas à constater que j'étais à la tête de matériaux infiniment plus composites que je ne l'avais escompté. Je dus aussi accepter que mon passé de professeur à El Atillo représentait à la fois une ressource indéniable pour mener à bien mes entretiens, mais qu'il m'inscrivait dans un rôle d'observateur critique non seulement du passé mais aussi des événements auxquels j'assistai, et qu'on me demandait de juger et d'apprécier parfois dans leurs moindres méandres.

\section{Diversité des témoignages et des témoins}

$\mathrm{Si}$, dans mon désir un peu naif de construire une série de récits autobiographiques, j'avais certes immédiatement entrevu que ceux-ci seraient d'inégale valeur d'un point de vue "documentaire » et «narratif », j'étais loin d'imaginer qu'ils seraient aussi hétérogènes. Certains récits, m'avait-il semblé, mettraient inévitablement en lumière des événements particulièrement importants, tandis que d'autres ne révèleraient que des faits plus anecdotiques, ou moins significatifs. Les multiples personnes interrogées feraient preuve de capacités à raconter bien évidemment inégales. Ces récits, dans leur diversité et leur hétérogénéité, permettraient une meilleure compréhension de cet ensemble que je souhaitais étudier : les guérilleros miskitus et leurs proches durant les années de guerre civile. Aussi était-ce fort de cette certitude qu'il m'était apparu comme indispensable de multiplier l'enregistrement de ces récits, de manière à constituer un corpus le plus complet possible, en suivant les indications de ces informateurs hors-pairs qu'étaient pour moi Mono et Wari. Et $j$ 'avais cru que ces entretiens constitueraient autant de pièces, certes différentes par la forme mais assez semblables sur le fond : j'avais la quasi certitude qu'elles n'en composeraient pas moins une "mosä̈que ", selon la formule d'Howard Becker ${ }^{12}$, c'est-à-dire un ensemble de données peu ou prou susceptibles du même type d'analyse. J'étais en revanche resté passablement aveugle à la multiplicité des registres de l'expression, comme aux visées émi-

12. Voir Becker H., introduction à la réédition de Shaw C.R., The Jack-Roller: A Delinquent Boy's Own Story, Chicago, University of Chicago, 1966. 
nemment diverses qui sous-tendaient ces «prises de parole ». Sans doute avais-je saisi d'emblée l'importance des variations parfois abyssales de mes conversations avec les uns et les autres, mais je m’égarai dans un premier temps en indexant de façon un peu mécanique ces variations aux différents degrés d'intimité, de familiarité, ou encore de sympathie, que j'entretenais avec mes interlocuteurs.

Je pris conscience de cette hétérogénéité foncière des témoignages que je commençai à recueillir lors d'une conversation sur l'Eglise morave avec Mono, dans le temple de sa communauté, à la veille de l'action de grâce. Me donnant à comprendre le fonctionnement de sa congrégation, me faisant visiter le temple et assister aux offices et rencontrer le pasteur, il me demanda en retour mon avis sur la religion et m’interrogea en détail sur mon athéisme. Ce ne fut qu'après que je lui eus expliqué que mon absence personnelle de foi ne me conduisait nullement à mépriser ses croyances et ses pratiques religieuses, ni à déconsidérer les expériences miraculeuses survenues pendant la guérilla, qu'il entreprit de me raconter certains des miracles survenus pendant la guerre. Il me fit ensuite comprendre tout l'intérêt que revêtait pour lui mon enquête sur la guérilla, et plus généralement sur l'histoire de la Moskitia, en m’offrant différents documents réunis à mon intention. Il me fit alors présent de plusieurs manuels, reçus à l'occasion d'un entraînement avec les troupes spéciales aux Etats-Unis, et de cassettes préparées à mon intention. Il avait ainsi non seulement enregistré des contes d'Asang, dits par son oncle Tio Benito, mais presque tous les débats de l'Assemblée du Conseil des Anciens qui s'était tenue à Puerto Cabezas, plusieurs jours durant, en février 1998, et qui à bien des égards rappelait l'Assemblée de Rus Rus ${ }^{13}$. Je saisis à cette occasion tout l'intérêt que Samuel portait à ma recherche, sa volonté de m'apporter son concours mais aussi le fait que cette double attitude était fonction de sa volonté de me voir en retour engager un véritable dialogue sur ses actions et ses croyances, tant passées que présentes. Il voulait certes que sa voix soit entendue, que ses dires soient pris en considération dans mon enquête ; mais il voulait d'une certaine façon mener sa propre enquête sur son passé comme sur celui des siens, et ce dans une manière de dialogue avec l'étranger que j'étais. Et, à bien des égards, le modèle de l'échange à la fois amical et intellectuel qu'il entendait avoir avec moi était celui que nous avions commencé à nouer à El Atillo.

Le voyage que nous fîmes ensemble pendant quelques jours, tout d'abord à Waspam puis à Bilwaskarma, au lendemain de l'ouragan Mitch et en pleine crise des rearmados ${ }^{14}$, acheva de me faire prendre conscience de la singularité

13. Communauté de la Moskitia hondurienne où les partisans de la Reforma convoquèrent en septembre 1985 une assemblée réunissant guérilleros et exilés pour décider du futur de la guerre ; voir Bataillon G., "Guerre et assemblée, découverte et apprentissage de la démocratie chez les Miskitus nicaraguayens (1981-1988)», op. cit.

14 . Soulèvement armé dirigé par plusieurs anciens guérilleros démobilisés qui survint en 1998. 
de son attitude. Je fus cette fois non seulement sommé de formuler mes jugements sur le passé, ou sur des manières de voir qui pouvaient me rapprocher ou m'opposer à lui, mais comme à l'époque d'El Atillo, d'exposer mes vues sur le présent de manière à tenter d'apprécier avec lui la complexité d'une situation. La situation régionale était en effet passablement tendue. L'ouragan Mitch avait entraîné une crue sans précédent du Rio Coco qui avait emporté toute une série de villages. Les autorités centrales paraissaient se soucier avant tout des désastres survenus dans la partie pacifique du pays. Les polémiques faisaient rage sur la réalité des destructions sur le Rio Coco et la nécessité d'apporter, ou pas, des secours aux villageois sinistrés. Le contexte régional était d'autant plus compliqué, que d'anciens guérilleros miskitus, les rearmados/réarmés, prétextant à juste titre le non-respect des promesses qui leur avaient été faites par le président conservateur Arnoldo Aleman lors de sa campagne électorale, barraient, depuis déjà plusieurs semaines avant l'ouragan, la route de Puerto Cabezas à Waspam, et parfois celle qui allait en direction des mines et de Managua. Ne se contentant pas de dévaliser et de rançonner les voyageurs, ils incendiaient parfois les véhicules, brutalisaient les chauffeurs et les passagers. Les véhicules ne pouvaient donc plus circuler, et l'acheminement des secours vers Waspam était paralysé. Forts de cette aubaine, les rearmados se montraient de plus en plus entreprenants, de manière à monnayer au prix fort leur adieu aux armes. Si le prétexte de notre voyage sur le Rio fut de constater les ravages occasionnés par la crue et de rencontrer d'anciens participants au séminaire de El Atillo, dont le maire de Waspam, quelques-uns de ses assesseurs, et plus encore Tigre 17/ Manuel Cunningham, le but du voyage fut aussi très politique. Mono et plusieurs autres membres de YAAD, dont Tigre, considéraient que les rearmados étaient très largement manipulés par les militaires sandinistes, comme par certains commerçants sandinistes en collusion avec les narcos. En aidant matériellement les rearmados, les uns en fournissant armes et uniformes, les autres de l'argent et de la nourriture, tous démontraient au nouveau gouvernement libéral d'Arnoldo Aleman et à ses représentants locaux au sein du Conseil régional, qu'il fallait compter avec eux. Ils signifiaient très clairement qu'il ne saurait être question de réduire certains de leurs passe-droits, notamment en matière de commerce illicite de cocaïne, d'armes, ou de bois précieux coupés illégalement. Mieux, en contrôlant largement certains des chefs de file des rearmados, ils se donneraient éventuellement par la suite le beau rôle, soit qu'ils les obligent à négocier, soit qu'ils leurs coupent les vivres, soit qu'ils les écrasent militairement ! Mono avait presque immédiatement dénoncé cette collusion et ce jeu de dupe lors d'une de ses émissions hebdomadaire à radio Miskut. Il avait aussi appelé les rearmados à cesser leurs barrages, à mettre fin à ce qu'il appelait non sans raison des "actes de banditisme ", et à entrer ensuite au plus vite en négociation avec le gouvernement central. Cette attitude courageuse lui avait valu, en retour, des menaces de mort de la part d'un des chefs rearmados. Passablement inquiet du tour pris par les événements, Mono entendait donc se rendre à 
Waspam, rencontrer certains des insurgés qu'il connaissait depuis les années 1980, les ramener à la raison et, ce faisant, casser cette dynamique qu'il jugeait parfaitement dommageable pour la région.

Nous nous rendîmes donc à Waspam en autobus pour aller ensuite en taxi collectif à Bilwaskarma rencontrer Tigre et certains des rearmados. Si j'interviewai bien évidemment Tigre et Mono sur leurs trajectoires pendant la guerre, ces conversations furent entrecoupées d'échanges sur l'actualité la plus immédiate. A Waspam, nous avions vu les sinistrés demander une aide qui n'arrivait pas, et nous avions pu avoir un aperçu tout à fait édifiant sur l'impréparation et le cynisme du maire, avant tout soucieux de détourner toute aide à son profit. Nous avions enfin été les témoins du renouveau du mythe de la frégate anglaise. En effet la seule aide apportée aux sinistrés du Rio Coco avait été celle dispensée par l'équipage d'un vaisseau porte-hélicoptères hollandais en manœuvre dans la Caraïbe. Les Hollandais avaient remonté le fleuve en crue et évacué certains malades pour les soigner à bord de leur navire. Mais, pour presque tous mes interlocuteurs, ces marins n'étaient nullement des Hollandais, mais bien évidemment des Britanniques. Je fis aussi la connaissance des rearmados. Tout d'abord de façon un peu tendue, quand nous passâmes l'un de leurs barrages routiers sur la route de Puerto Cabezas à Waspam, puis de façon extrêmement tranquille, sur la route de Waspam à Bilwaskarma. J'assistai enfin, sans piper mot, à une réunion avec eux chez Tigre.

Durant tous ces moments, je réappris non seulement à voir et à juger en parallèle avec Mono, comme je l'avais fait à El Atillo, mais à confronter nos expériences et nos points de vue sur la situation, de façon systématique. A bien des égards, les sentiments qui avaient été les nôtres, les jugements que nous avions portés sur les rearmados et sur le maire de Waspam, nous permirent à l'un comme à l'autre de jauger nos capacités respectives d'apprécier des événements et de les interpréter. S'institua ce faisant, non seulement un rapport d'égalité entre nous, et ce quelles que soient nos différences dont nous avions l'un et l'autre conscience. J'étais un universitaire français, il était un ancien guérillero miskitu devenu l'un des responsables administratifs de l'hôpital de Puerto Cabezas. Nous avions tous les deux pareillement voix au chapitre pour interpréter et juger du présent comme du passé. Se dessina aussi une expérience de la similitude entre nous deux. Nous ne nous contentâmes pas d'exposer nos opinions sur les événements, de les mettre en parallèle, mais nous les discutâmes. Et, dans ces échanges apparurent des modifications de nos points de vue respectifs. J'appris non seulement à l'écouter, mais à reformuler mes questions et mes jugements en prenant en compte son point de vue ; il apprit à faire de même, non seulement sur la situation présente, mais sur le passé. 
Cette modalité du dialogue qui fut le nôtre et le resta tout au long de l'enquête s'appuyait aussi sur la double volonté de Mono de devenir à la fois pasteur, c'est-à-dire de réaliser des études de théologie, et instituteur. Ce double désir, dont seul le second fut réalisé dans les années qui suivirent, pesa sans doute de façon décisive dans notre manière de dialoguer. En 1984, Samuel avait été de ceux qui avait le plus participé aux discussions que j’avais eues avec les cursantes de El Atillo sur ce qui me semblait être les impasses et les aspects les plus critiquables de leurs façons de mener la guerre. Il avait aussi été un des plus réceptifs à mon projet d'écrire sur l'histoire de la guérilla. Il m'avait ainsi raconté comment, plus que d'autres, il s'était approprié les cours et les discussions sur l'histoire des guérillas, comment il les avait utilisés au moment des pourparlers préalables à l'assemblée de Rus Rus, notamment lors de rencontres avec des commandants en délicatesse avec Brooklyn Rivera, rencontres et alliances dont devait sortir la Reforma. Si cette capacité à faire sien un enseignement et à le réutiliser à sa manière avait bien évidemment tenu à son génie propre, comme à celui de ses associés, ce geste ne s'en inscrivait pas moins dans toute une tradition liée à l'expérience de l'évangélisation par les moraves. La situation des jeunes commandants durant le second semestre 1984 n'était pas sans parallèle avec celle des Miskitus aux débuts de l'évangélisation. Aux débuts de leur conversion, les Miskitus avaient pu tabler sur des visites très régulières des missionnaires allemands installés à Bluefields. Puis, à la suite de retournements de la conjoncture politique due à la victoire de Zelaya (1900), qui ordonna la fermeture des écoles moraves, et à une série d'ouragans (1906, 1908 et 1909) qui détruisit la goélette permettant aux missionnaires de se déplacer, les premiers convertis furent livrés à eux-mêmes. Comme le note l'historien des moraves miskitus John Wilson, cette époque fut paradoxalement celle d'une expansion durable de l'évangélisation. Des pasteurs et les laïcs eurent à cœur de remplacer les missionnaires absents, ce fut sans doute là que «surgit pour la première fois l'idée d'une Eglise nationale 15 ». De même, au milieu de l'année 1984, les jeunes commandants miskitus, après avoir reçu les deux années précédentes une aide militaire substantielle des Etats-Unis, et avoir pu converser souvent très longuement avec des observateurs étrangers (journalistes, cinéastes ou anthropologues) qui multiplièrent plus ou moins ouvertement les critiques à l'égard de certaines de leurs actions, se virent peu ou prou livrés à leur sort. En effet, l'aide létale fut pratiquement suspendue, tandis que beaucoup d'entre eux, comme Mono, furent renvoyés dans des zones de guerre, c'est-à-dire à distance de tout contact ou de toute personne susceptible d'inciter à une analyse critique de l'action. A l'image de leurs ancêtres, qui s'étaient appropriés un certain nombre de dispositifs des moraves et qui avaient ainsi poursuivi le travail d'évangélisation, Mono et plusieurs autres commandants avaient fait de même, cette fois non dans une visée religieuse mais politico-militaire. Ils avaient mis à profit le flottement des guérilleros,

15. Wilson J., Obra morava en Nicaragua: trasfondo y breve historia, Managua, Unión, 1990, p. 122. 
chaque jour davantage livrés à eux-mêmes, pour procéder à une réorganisation de la guérilla en s'appropriant des habitudes réflexives renforcées par leurs contacts avec des observateurs étrangers ou des instructeurs militaires de tous bords, séjournant plus ou moins longtemps à leurs côtés. Le souci de dialogue, manifesté par Mono, ainsi que sa volonté de réaliser cette enquête avec moi, s'inscrivaient dans cette logique d'un métissage intellectuel actif promu par les moraves et valorisé par toute sa tradition familiale. Sa double aspiration au pastorat et au professorat en était le signe le plus évident.

Ces premiers échanges avec Mono me convainquirent en outre de la nécessité d'élargir la période que je m'étais proposé d'étudier au départ : les années de la révolution sandiniste et de la guerre. En effet, si la chronologie rédigée au sortir de la guerre par Santiago s'ouvrait sur la fondation d'ALPROMISU en 1973, bon nombre de mes interlocuteurs, dont Mono, inscrivaient leurs récits dans une temporalité beaucoup plus longue. Ils reliaient ainsi leurs propres histoires non seulement à celles des années 1950-1960, à la Seconde Guerre mondiale, mais aussi aux années 1920-1930, aux guerres du «vieux » Sandino et, parfois, à l'époque de l'évangélisation, à la charnière du $\mathrm{XIX}^{\mathrm{e}}$ et du XXe siècles. Les sœurs Bob m'avaient ainsi présenté leur oncle pour qu'il témoigne des méfaits des hommes de Sandino; Benigno avait, à l'inverse, évoqué la collaboration volontaire de son père avec ce dernier. Ces deux manières de s'ancrer dans les années 1920-1930 rappelaient les réfugiés que j'avais connus en 1984 qui, eux, mentionnaient l'assassinat du pasteur Bregenzer à Musawas, puis peu après l'exode de beaucoup de villages miskitus, dont Asang, sur le Rio Patuca, pour échapper à l'emprise de Sandino et de ses hommes. Mono m'expliqua ainsi, lors de l'une de nos conversations, quelles avaient été les discussions dans les familles, notamment la sienne, lors du triomphe des sandinistes en 1979. Si les plus jeunes, et les adultes nés au lendemain des années 1930, avaient dans l'ensemble eu a priori des sympathies pour le renversement de Somoza et pour les promesses de la révolution du 19 juillet, les plus âgés les avaient mis en garde contre ces guérilleros qui se revendiquaient de Sandino, le «bandit des Segovias ». De même, quand lui ou d'autres évoquaient l'histoire de leurs communautés, de leurs parents et de leurs grands-parents, ils remontaient volontiers jusqu'à l'arrivée des missionnaires, c'est-à-dire à l'époque de l'évangélisation des Miskitus et à la fondation des premiers villages. Tous narraient, plus ou moins systématiquement, leurs histoires propres, ou celles de leurs familles, en fonction d'une temporalité fixée par l'Eglise morave : les «temps obscurs » d'avant l'évangélisation, évoqués dans les contes et légendes; les débuts de l'évangélisation sous la houlette des missionnaires allemands; les «temps difficiles » des guerres de Sandino et la reprise de l'évangélisation avec l'aide des missionnaires nord-américains; puis l'époque de l'autochtonisation (1949-1974) et de l'indépendance de l'Eglise, qui allait de pair avec l'insistance sur la « lutte indigène ». L'influence morave ne se faisait pas seulement sentir dans ces temporalités indissociables et com- 
plémentaires, mais aussi dans le style même des mises en récit de ces différents épisodes. Sans doute l'ordre chronologique des épisodes de l'histoire de l'Unitas Fratrum était-il bouleversé. A la différence des moraves européens et nord américains, les Miskitus avaient commencé par connaître des « temps obscurs » avant que la lumière des moraves n'arrive jusqu'à eux, tandis que Jean Hus et ses disciples avaient connu, comme les premiers chrétiens, le martyr et les persécutions. Les «temps difficiles » n'étaient venus qu'après l'arrivée des « lumières » de l'évangélisation, à l'époque de Sandino, où l'Eglise avait eu son martyr en la personne du pasteur Bregenzer, puis avec l'arrivée au pouvoir du Front sandiniste et les persécutions qui s'en étaient suivies. Ces inversions évidentes ne sauraient dissimuler la profonde parenté entre les manières de raconter et de mettre en scène à la fois les moments heureux et les moments dramatiques de la vie des communautés et des individus. La simplicité dont étaient empreints les récits en constituait un des traits les plus frappants. Nul pathos, nulle emphase, la dramaturgie reposait sur une façon de s'exprimer a minima, parfois même avec une certaine sécheresse. De ce point de vue, le ton était toujours autre que celui des récits miskitus «traditionnels ", les contes et les légendes racontant des épisodes toujours antérieurs à l'arrivée des moraves ayant souvent une tonalité beaucoup plus ironique, évoquant l'amour et la sexualité, la chasse et la nature, des êtres surnaturels : autant d'éléments totalement absents des récits que je recueillais. Les récits des épisodes de la conversion comme ceux de la fondation des villages n'étaient pas sans rappeler, par le ton comme par le style de la mise en scène, les récits de la fondation de Herrnhut. S'y faisait jour une même idée de la grâce et de la révélation. De même, l'histoire des persécutions (qu'il s'agisse des années 1920-1930 et des heurts avec les hommes de Sandino, de l'incendie des villages en 1982, des arrestations et des assassinats commis à cette époque par l'armée, ou enfin de l'exil de milliers de Miskitus au Honduras) était-elle racontée dans des termes qui évoquaient irrésistiblement certains temps forts des épreuves qu'avaient connues hussites et moraves. On prêtait ainsi à Tomas Borge, le ministre de l'Intérieur sandiniste, des paroles ("je suis prêt à tuer tous les Miskitus pour en finir avec la Contra ») qui étaient l'exact reflet des mots attribués à l'archiduc Ferdinand, à la veille de la guerre de trente ans et de l'épisode de la Montagne blanche : "mieux vaut un désert qu'un pays plein d'hérétiques ». La tuerie de Leimus, la destruction des villages, les deux exodes vers le Honduras, autant de moments clés qui, là encore, étaient relatés comme en écho aux persécutions organisées par les jésuites en Bohême au lendemain de la défaite de la Montagne blanche, à la fuite vers Herrnhut, ou à l'exode dans le Nouveau Monde ${ }^{16}$. Dernier parallèle avec les récits moraves : la capacité à se mouvoir dans une histoire cumulative, conçue comme le récit d'une lutte

16. Ces comparaisons sont on ne peut plus frappantes si on les rapporte non seulement à l'histoire des moraves, mais aussi à la façon dont celle-ci est présentée dans le petit livre qui sert à l'édification des fidèles : Schattschneider A.W., Durante quinientos años. Una historia de la Iglesia morava, trad. par T.A. Good, Bethlehem (Pennsylvania), Departamento de Misiones Extranjeras de la Iglesia Morava en América, 1961, surtout chap. III et IV, pp. 28-40. 
pour la liberté et les lumières. En effet, les récits faisaient s'enchaîner des épisodes conçus comme autant de moments liés les uns aux autres au cours desquels les acteurs accumulaient des savoir-faire et des expériences. Si les persécutions n'étaient jamais absentes de ces mises en récits, elles étaient autant de moments où l'on se confortait dans sa foi, et ce dans une tradition très chrétienne, mais aussi l'occasion de déployer une dialectique laïcisée mettant en avant l'idée d'une accumulation d'expériences analysables, dès lors plus aisément surmontables par la suite. Se faisait jour l'idée d'apprentissage et d'acquisition de savoirs nouveaux dans les luttes et les épreuves. Ainsi, quel qu'ait été mon désir de borner les termes de mon enquête au dernier quart du $\mathrm{XX}^{\mathrm{e}}$ siècle, il m'apparut indispensable de laisser mes interlocuteurs déployer leurs récits dans un temps qui courait sur tout ce siècle, voire sur la fin du précédent, quitte à privilégier par la suite l'analyse de la période qui avait au départ retenu mon attention.

Ce voyage à Waspam et à Bilwaskarma fut, en outre, l'occasion de mieux saisir les différences entre mes relations avec Mono - relations qui conditionnaient sa manière de témoigner, tout comme la mienne de susciter son récit et celles qui pouvaient me lier à d'autres personnes dont je sollicitai et obtint des « autobiographies modèles ", telles mes anciennes connaissances de la guérilla ou certains de leurs compagnons des années de guerre, autant de personnalités très différentes. Tigre était toujours un reître formé au sein de la Garde nationale de Somoza et, sur ce point, sa participation à la Reforma, puis son travail avec le Centro de Investigacion y Documentacion de la Costa Atlantica (CIDCA) étaient loin de l'avoir complètement métamorphosé. Il avait le verbe haut et il n'était pas homme à se laisser menacer impunément. Kenneth était au premier chef un médecin-chirurgien, dont le coup d'œil, le sens du diagnostic et l'humanité en faisaient un praticien de génie doublé d'un intellectuel plein de finesse. D'autres encore étaient à bien des égards des exemples de «traumatisés » de guerre qui parfois jouaient fort bien de cette catégorie pour pratiquer avec efficacité un "familial amoralism 17 » souvent assez douteux. D'autres enfin, tel un ancien second de Mono devenu comptable, ou encore Julian, chauffeur de taxi préoccupé par son rôle au sein de sa congrégation, s'étaient fort bien réadaptés à la vie civile. Par-delà ces disparités, tous partageaient une même soif de réflexion et acceptèrent de très bonne grâce mes demandes de prendre sous leur dictée leur récit de vie. Bien que Tigre, Felix ou Tata aient été plus en retrait dans cette démarche que d'autres comme Adam, Kenneth, Julian ou Santiago, tous, sans aller aussi loin que Mono dans le souci de s'interroger sur le passé et le présent, n'entendaient pas seulement donner leurs témoignages, mais bel et bien les discuter avec celui qui les recueillait. Tous avaient également envie de discuter de la situation du moment; discussions qui alimenteraient en retour leurs remémorations. Tous

17 . J'emprunte cette notion à Banfield E., The Moral Basis of a Backward Society, Glencoe, The Free Press of Glencoe, 1958. 
enfin souhaitaient savoir ce que je ferai de leurs paroles et se montraient en outre prêts à me fournir certaines de leurs archives. Les uns me donnèrent des photos, d'autres regrettèrent d'avoir jeté de vieux documents de la guérilla, jugés en trop mauvais état pour être conservés. Ils me firent tous par la suite toujours bon accueil et furent, comme moi, friands d'échanges, particulièrement sur les jeux politiques du moment. Mais, à l'exception de Samuel, aucun n'aspira à une sorte d'entrelacement de ses réflexions avec les miennes, ni à un dialogue sans bornes. S'ils témoignèrent et réfléchirent sur leurs trajectoires comme sur le présent, s'ils suscitèrent ma réflexion et m'invitèrent au dialogue et au commentaire, ces échanges n'allèrent pas jusqu'à tenter de renverser les points de vue. Ils ne se firent que rarement questionneurs systématiques sur ma manière de voir et d'évaluer les phénomènes. Il fut d'autre part entendu que nos conversations trouveraient un terme après un premier tour d'horizon. Ils ne s'inscrivirent pas comme Samuel dans un dialogue et un travail de réflexion en droit infini, voué à l'indétermination et à la reprise, même s'il était voué à être interrompu pour pouvoir fixer des états de la réflexion sous formes d'articles ou de livres. L'horizon d'attente dans lequel s'inscrivaient nos rencontres se situait dans le prolongement de mon rôle de professeur à El Atillo. Ils m'apportaient des informations comme j'en avais apporté en 1984 aux guérilleros, à l'époque de mes cours, sans pour autant que les barrières entre auditeur et récitant soient brouillées ou mises en question. Par delà des rencontres dans une manière d'égalité et de similitude, lors de moments de réflexion commune, il n'y eut pas ce souci, partagé avec Mono, d'une réflexion au long cours mené de concert.

Ce séjour me permit enfin d'isoler une troisième modalité du témoignage propre à des personnes le plus souvent âgées, comme Rogelio Bob, Nicodemo Serapio, Laurita et Tillith Mollins. Elles s'inscrivaient dans la logique d'un témoignage où le souci de l'exemplarité l'emportait indéniablement sur celui de la réflexivité critique. Nul doute qu'en apparence, leurs façons de narrer les histoires n'aient été des plus variées. Les uns et les autres ne mettaient que fort rarement l'accent sur les mêmes évènements et ils les narraient de façons assez différentes. Rogelio Bob, bien que peu ou prou du même âge que les Mollins, insista avant tout sur les années des guerres de Sandino et ce qu'il avait alors connu. Les Mollins n'en soufflèrent pas mot. En revanche, Laurita mit au centre de sa perspective l'histoire de l'école d'infirmière de la mission morave de Bilwaskarma, "la première école d'infirmière de tout le pays ", puis son rôle comme infirmière sur le Rio Coco. Nicodemo Serapio fut, quant à lui, fort disert sur son moment de gloire, l'époque des premiers combats entre les guérilleros miskitus et les sandinistes, et beaucoup plus discret, pour ne pas dire muet, sur le reste de sa vie. Par-delà leurs discordances, ces narrateurs s'inscrivaient pourtant dans une logique commune, celle de récits de vie exemplaires mettant en scène un passé qu'ils souhaitaient transmettre aux générations futures. Dans des modalités assez semblables à celles mises en lumière par 
David Brumble dans son étude des autobiographies indiennes d'Amérique du Nord, ils se livrèrent à ce que ce dernier a nommé des «récits de coups»18. Ils ne relatèrent que ce qu'ils considéraient comme leurs hauts faits, c'est-à-dire à leurs yeux les épisodes les plus saillants de leurs vies. Jamais ils n'eurent le projet d'une narration faisant place à la totalité de leurs expériences et moins encore à des interrogations sur celles-ci. Ces récits furent immanquablement centrés sur certains moments jugés particulièrement significatifs de leurs trajectoires, dont les narrateurs me firent des sortes de listes plus ou moins longues et détaillées. Que ces moments aient été marqués du sceau du malheur et de la persécution comme le récit des brutalités des hommes de Sandino à l'encontre des Miskitus du haut Rio Coco chez Rogelio Bob, ou bien que, ces moments aient été perçus sous le signe d'un succès mondain ou d'un bonheur privé, comme dans le récit de Laurita Mollins, un même souci de ne faire place qu'à certains événements jugés dignes d'une mise en mémoire s'affirma chaque fois. Le reste ne comptait à leurs yeux que peu ou pas.

Tous ces témoins eurent une autre caractéristique commune, qui pesa de façon décisive sur leurs capacités à prendre la parole. Ils se pensaient et étaient considérés, à l'époque où je les interviewai, comme des vieillards dont il fallait recueillir les dires sans tarder. Eux et leurs proches avaient à la fois le sentiment de leur fin prochaine et du caractère souvent exceptionnel de ce qu'ils avaient l'habitude de raconter à leurs proches, d'où l'idée de fixer ces dires par l'écrit. La préparation de ces entretiens et les conversations que j'eus avec eux, me convainquirent qu'ils s'étaient installés, parfois de leur propre chef, parfois à l'initiative de leurs proches, dans des positions assez semblables à celles des conteurs traditionnels, dont les folkloristes publiant la revue Tiniska collectaient contes et légendes. En s'adressant à un étranger, perçu comme un écrivain-journaliste, ils aspirèrent à figurer dans un livre destiné à être lu par un public plus large que celui des Miskitus. Cette aspiration à voir leur parole s'imprimer dans un futur livre fut couplée à la volonté d'accéder à ce potentiel réflexif élémentaire de ce que Jack Goody a nommé "la raison graphique », aux dépens de la seule transmission par la voie orale, désormais jugée insuffisante pour ancrer dans la mémoire collective certains faits, certains récits. Cette volonté de s'inscrire dans un livre fut aussi considérée comme une opportunité d'accéder à une dignité nouvelle. Tous ces narrateurs y virent une possibilité d'inscrire leurs vies à hauteur, non seulement de celles des héros de la mémoire collective miskitue (le cacique Miskut, les frères Aimapaya ou Kuridam) comme de ceux de l'histoire nicaraguayenne (Sandino, Carlos Fonseca Amador ${ }^{19}$ ou Pedro Joaquin Chamorro ${ }^{20}$ ), mais aussi au niveau des célébrités mondiales et des personnages évoqués par les médias. Mon livre ou

18. Brumble D., American Indian Autobiography, Berkeley, University of California Press, 1990.

19. Fondateur du Front sandiniste de libération nationale.

20. Originaire d'une famille conservatrice de Granada, Pedro Joaquin Chamorro fut le directeur du quotidien La Prensa, le grand quotidien d'opposition à Somoza. Son assassinat (le 10 janvier 1978) fut un des moment clé des mobilisations populaires contre la dictature de Somoza. 
mes articles à venir furent, par ailleurs, perçus comme autant de possibles contrepoids aux brochures du FSLN (Front Sandiniste de Libération Nationale) notamment au livret d'alphabétisation des sandinistes, $E l$ Amanecer del Pueblo, qui avait constitué une véritable vie des héros et des martyrs sandinistes. Ils furent aussi considérés comme des prolongements venant corriger et amender des publications comme celles patronnées par l'antenne nicaraguayenne du Nature Conservancy (TNC), Wani ou Tinniska, qui dans l'ensemble n'évoquaient à aucun moment les périodes de guerre, qu'il s'agisse des affrontements des années 1920-1930, ou plus encore des années 1980. Ce désir de voir leurs dires imprimés fut une manière de reprise laïcisée de la tradition des moraves - déjà évoquée -, soucieux de faire connaître dans leurs publications les gestes de certains de leurs fidèles. Ce goût pour l'écrit fut d'une certaine manière un doublet de la revendication des anciens combattants de la guérilla, et tout spécialement des membres de YAAD et des artisans de la Reforma, d'ériger un monument aux morts miskitus à Waspam. Les épisodes narrés par ces personnes âgées m'apparurent comme autant d'équivalents des noms dont les plaques du futur monument aux morts portaient la trace. Ces récits et ces noms étaient autant de supports ou de marques de la mémoire collective sur lesquels s'édifieraient des réflexions et des discussions sur l'histoire des Miskitus. Dans le rapport qu'entretenaient les vivants les plus jeunes sur le passé, qu'ils aient ou non participé aux mobilisations des années 1980, ces traces et ces noms des morts me parurent être des repères historiques au même titre que l'étaient les pierres portant des pétroglyphes du Rio Coco que les Miskitus aimaient à montrer comme preuves de l'ancienneté de leur civilisation et des aptitudes artistiques de leurs ancêtres.

J'appris parallèlement à repérer tout un nouveau discours indianiste, dont les prototypes étaient pour moi les discours des membres du Conseil des anciens et de leurs assesseurs. Je rencontrai ceux-ci au lendemain d'un incident qui les avait opposés au président de la République, Arnoldo Aleman, lors de l'un de ses déplacements officiels dans la région. Celui-ci avait en effet exigé que le seul drapeau présent sur les édifices officiels soit celui du Nicaragua et que soit abaissé celui de la Moskitia, à bien des égards copié de l'Union Jack, qu'avaient fait hisser les membres du Conseil des anciens. Ceux-ci s'entretinrent longuement avec moi de cet incident, dans lequel ils voyaient une marque de dénégation raciste de l'ancienneté de la nation miskitue. Si je reconnus sans peine le racisme indéniable des Nicaraguayens hispanophones vis-à-vis des Miskitus, je ne cachai pas un certain scepticisme quant à l'idée de «l'ancienneté du sentiment national miskitu». J'eus alors droit à la formulation d'un récit des origines de la nation miskitue extrêmement élaboré. Ce récit obéissait incontestablement à une mise en scène particulièrement rigide qui faisait la part belle à quelques-unes des thématiques à tout le moins schématiques de la commémoration de 1492 : la conquête était assimilée à un " génocide "; l'accent était ensuite mis sur «l'exploitation coloniale », tandis que tous les pro- 
cessus de métissages étaient purement et simplement gommés ; on affirmait a priori l'existence "d'une harmonie entre les peuples autochtones et la Nature »; on vantait l'existence d'une «démocratie tribale » et de relations pacifiques entre les différents groupes amérindiens. Ce nouvel indianisme ne conduisit pas simplement mes interlocuteurs à d'invraisemblables reconstructions historiques, mais aussi à réélaborer leur histoire personnelle en fonction de ces impératifs. Les aléas de l'histoire et des événements survenus au cours du dernier demi-siècle, les répercussions de ceux-ci sur les destinées personnelles de mes narrateurs, ne trouvèrent de place qu'à la condition d'être autant d'éléments s'inscrivant sans accroc dans ce nouveau roman des origines de la nation miskitue. On m'évoqua une vie dans des villages où l'Eglise morave et son clergé étaient purement et simplement absents. On me raconta sans sourciller le " passé immémorial » du Conseil des anciens, alors que mes interlocuteurs savaient pertinemment comment celui-ci avait été créé par Fagoth à des fins parfaitement prosaïques, au début des années 1980. Ce faisant, celui-ci avait voulu donner un vernis d'indianité, façon ligue des Iroquois et Indiens de Western, aux réfugiés miskitus que leur protestantisme piétiste suffisait à discréditer comme Indiens aux yeux d'une opinion publique internationale plus avide de clichés que d'analyses subtiles. Il avait aussi voulu créer une organisation représentative des réfugiés à sa dévotion, et dont il puisse se prévaloir dans ses bagarres avec les représentants du HCR qui n'entendaient nullement le voir transformer les camps de réfugiés en sanctuaires de la guérilla. Du coup, les combats de nombreux leaders des communautés en exil - combats appuyés par les guérilleros réformateurs, pour à la fois évincer les métis hispanophones mis en place à la tête du Conseil des anciens par Fagoth, et transformer cette institution en une manière de conseil informel de l'ensemble des communautés mayangnas, miskitues et créoles - furent systématiquement passés sous silence. Toutes les tensions qui avaient pu exister entre les Miskitus et les Mayangnas durant la guerre, les premiers étant accusés à juste titre par les seconds de les avoir rendus corvéables à merci, devinrent de même indicibles. Mes différents interlocuteurs avaient participé à l'assemblée de Rus-Rus et l'un d'eux avait pris part aux discussions ayant mené à la paix de Yulu. Les débats dans lesquels ils s'étaient engagés représentaient des faits qui ne pouvaient être que gommés au profit de déclarations essentialistes visant à limer toutes les aspérités de processus socio-politiques dont ils avaient été parties prenantes. Ce goût pour la reconstruction politico-historique s'adossa à un souci de mettre en avant des explications générales, presque toujours détachées de leurs expériences réelles ou de faits avérés et vérifiables. Ainsi en allat-il de leurs propos sur la corruption des élus aux conseils municipaux ou aux conseils régionaux. Enfin, en filigrane, je sentis chaque fois une série de préoccupations parfaitement prosaiques qui consistaient à évincer à leur profit toute idée de représentation et de délégation. Quoi de plus simple et de profitable pour eux que de se poser en représentants " naturels » des communautés! Ils n'avaient dès lors plus à affronter ni l'incertitude d'un processus électoral à 
bulletin secret, ni le pouvoir de sanctions des électeurs. L'idée qu'ils incarnaient les communautés leur permettait en outre de justifier leurs prétentions à occuper différentes positions prestigieuses et éminentes, de réclamer pour eux et leurs assesseurs certaines prébendes allant de pair avec ces positions, ou de pouvoir contrôler toute une partie des ressources affectées au développement de la région. Sans pour autant céder à une opposition chimérique entre des discours reflétant au premier chef des intérêts dûment constitués, donc marqués par le mensonge idéologique, et d'autres mettant en scène des expériences à distance de toute visée pragmatique, donc purs de toute dissimulation d'intérêts particuliers, je n'en appris pas moins à prendre le recul nécessaire face à des interlocuteurs comme les membres du Conseil des anciens. Je ne pus donc faire l'impasse sur les méandres d'une idéologie indianiste en plein essor dans différents milieux sociaux de la Moskitia, notamment chez des jeunes ayant eu accès à des études secondaires : mais je jugeai qu'il ne convenait pas pour autant de lui donner une primauté injustifiée. Si j'acceptai d'en recueillir quelques échantillons, je pris plus d'une fois la décision d'éconduire, parfois assez sèchement, ce genre d'interlocuteurs, et ce d'autant qu'ils cherchaient parfois à monnayer leurs confidences.

Je fis enfin l'expérience de l'impossibilité de certains entretiens, tout spécialement avec les jeunes femmes que j'avais connues à l'époque de la guérilla. Beaucoup d'entre-elles avaient été mariées ou avait vécu maritalement durant ces années. Et assez banalement, celles-ci avaient été quittées pour des femmes plus jeunes, soit pendant la guerre elle-même, soit au lendemain de celle-ci. Aussi, quand plus de dix années après, je tentai de recueillir leur histoire, je constatai que mon amitié avec les anciens compagnons de certaines d'entre elles comme avec elles-mêmes, et la camaraderie amoureuse que j'avais entretenue avec l'une d'elle, nous mettaient dans une situation où ni elles ni moi n'arrivions à nous plier au jeu de rôle que supposait la construction d'un récit de vie. La difficulté ne résidait pas seulement dans l'impossibilité de m'isoler seul à seul pour converser. Nous nous retrouvions parfois pour prendre un café ou un soda dans le salon-véranda de l'hôtel de Doña Aurita. Il y avait plus profondément de leur part une véritable répugnance à relater des vies conçues pour une bonne part comme marquées par le sceau du malheur, et où les hommes étaient perçus comme des trompeurs ou des persécuteurs. Je ne pus donc accéder qu'à des récits très largement hachés et tournant court assez vite. En effet, dans la mesure où je souhaitais évoquer avec elles la façon dont elles avaient vécu non pas la guerre mais les années antérieures à celle-ci, il nous fut toujours possible d'évoquer leur enfance ou les années 1979, 1980, et 1981. Toutes me racontèrent ainsi très volontiers comment elles avaient fait partie d'un groupe de danse folklorique mis sur pied par une professeur qui avait été l'une des chevilles ouvrières de la renaissance de la culture miskitue, Herta Downs, groupe dont les activités avaient été relancées au sein de MISURASATA. Nous évoquions aussi les campagnes d'alphabétisation en espagnol, 
puis en miskitu, tout comme leur fuite au Honduras et leurs apprentissages du métier de guerillera. Mais dès que nous abordions les périodes où elles avaient rencontré leurs compagnons, celles de leurs maternités, puis ce qu'elles vivaient toujours, non pas comme des séparations mais comme des abandons de la part de leur conjoint, tous les entretiens tournèrent court d'une façon ou d'une autre. Ces évènements restaient des blessures toujours à vif, parfois plus de dix ans après les faits. Quelques-unes me contèrent d'ailleurs leurs difficultés à évoquer ces questions au sein de l'Eglise morave, lors de leurs examens de conscience annuels face aux anciens de la congrégation. Si celles-ci furent souvent intarissables et drôles sur ce qu'elles nommaient le côté «libidineux » de plusieurs anciens, beaucoup d'autres ne purent même pas évoquer la difficulté à parler de leur statut de filles-mères, tant il allait de pair avec une dégradation de leur condition aux yeux de toute une partie de l'Eglise morave. Ces difficultés à s'exprimer furent encore accentuées par mon impossibilité à construire un cadre adéquat pour accueillir leurs dires. Les quelques révélations que j'obtins s'accompagnaient incontestablement d'angoisses répétées et renouvelées, angoisses auxquelles je n'étais nullement préparé à faire face, n'étant d'aucune façon analyste ou psychologue. Je décidai donc très vite de cesser de tenter de recueillir de telles histoires de vies, me contentant de multiplier les conversations à bâtons rompus, presque toujours en groupe, dans des situations se prêtant le moins possible à de quelconques déstabilisations. Et dès lors qu'elles apparurent à deux ou trois devant moi, elles optèrent pour un ton infiniment plus libre, commentant souvent entre elles leurs "malheurs", me demandant comment de telles choses étaient vécues en France. De même, elles évoquèrent avec moi, souvent très en détail, les accords prosaïques qui sous-tendaient un jeu politique que beaucoup d'hommes passaient, eux, pudiquement sous silence, à la façon dont nous avions pu évoquer ces aspects dès l'époque de la guérilla, notamment à El Atillo ${ }^{21}$.

Vrai et faux.

Les multiples récits et les différentes informations que je pus recueillir me confrontèrent on ne peut plus clairement à la question de la véracité des dires de mes interlocuteurs et à celle de l'incontournable problème du recoupement de leurs récits. Il était évident qu'on ne me racontait pas tout, que les paroles étaient prononcées selon certains biais. On pouvait me mentir, soit par omission, soit en inventant, et ce pour garder le « beau rôle », l'acquérir, ou pour stigmatiser des rivaux, ce qui revenait peu ou prou au même. Il était à l'évidence des silences qui étaient de peu d'importance. En effet, certains domaines de la vie personnelle de mes interlocuteurs, notamment tout ce qui relevait de leur intimité, étaient très généralement passés sous silence. Dans la mesure où

21. On trouvera dans l'étude de Jules Falquet une analyse remarquable de ces phénomènes dans le contexte de la guérilla salvadorienne : Falquet J., " Division sexuelle du travail révolutionnaire : réflexions à partir de la participation des femmes salvadoriennes à la lutte armée (19811982) ", Cabiers des Amériques Latines, n40, 2002, pp. 109-128. 
mon propos n'était pas de parvenir à une description ethnographique des démarches amoureuses, du mariage et de la vie familiale des indigènes de la Moskitia nicaraguayenne, cela n'avait guère d'importance. En revanche, il était des omissions, des réécritures ou des mensonges qui pouvaient toucher à des sujets qui, sur le fond comme sur la forme étaient d'une importance capitale pour mon projet d'une anthropologie de la guérilla.

Les discussions suscitées par le témoignage de Rigoberta Menchu furent particulièrement éclairantes pour mon propos. J'étais en effet à bien des égards très souvent dans une situation assez semblable à celle d'Elizabeth Burgos, coauteur de Moi Rigoberta Menchu 22. Ce livre est à l'évidence une " autobiographie modèle ». Une jeune femme quiché, liée à un des groupes de la guérilla guatémaltèque, l'Ejercito Guerillero de los Pobres (EGP), interviewée en espagnol, raconte à sa manière sa trajectoire de vie et donne sa vision du monde au moment où elle est interrogée. Mais ses dires, comme l'avait établi un anthropologue américain David Stoll 23, reposaient sur d'indéniables travestissements de la réalité. Rigoberta Menchu n'était nullement issue d'une famille de paysans pauvres, mais au contraire d'une famille relativement aisée ; loin de n'avoir jamais pu accéder à l'école, elle avait aussi été brièvement scolarisée ; enfin sa politisation avait été plus tardive que ce qu'elle avait relaté. En effet, contrairement à ses dires, elle n'avait pas "accédé à la conscience " grâce à la participation de son père à la fondation du Comité d'unité paysanne (CUC) et à ses menées contre les grands propriétaires fonciers, ou encore à ses séjours dans les grandes plantations sur la côte pacifique. Sa politisation et son adhésion aux thèses de l'EGP avaient été postérieures à son exil au Mexique, après les assassinats de son père et de son frère. Le premier, Vicente Menchu, n'avait probablement pas joué le rôle de dirigeant paysan, en liaison avec le CUC, qu'elle avait peint dans ses mémoires; il se serait lié à l'EGP en pensant trouver en la guérilla un appui dans des conflits locaux l'opposant, entre autres, à sa belle famille. Enfin, elle n'aurait nullement assisté à l'assassinat de son frère, qui avait certes bien été exécuté par l'armée, mais dans d'autres circonstances que celles relatées dans son autobiographie. Tout l'intérêt de l'enquête de David Stoll avait été de permettre de saisir comment certaines représentations de la réalité avaient été valorisées par l'intelligentsia liée à l'EGP, et comment ces représentations avaient été tout à la fois imposées et intégrées par toute une partie de la paysannerie indigène, peu à peu acquise à la cause et aux visées de la guérilla. En effet, rien de plus significatif que ce gommage de conflits agraires opposant et mettant en rivalités des petits propriétaires microfundistes, par ailleurs parfois commerçants, les uns indiens, les autres métis, au profit d'une opposition binaire : microfundistes indigènes ou métis, voués au salariat temporaire dans les grandes exploitations agro-exportatrices versus latifundistes métis se pensant comme les descendants des conquista-

22. Burgos E., Me llamo Rigoberta Menchu, Mexico, Siglo XXI. 1983.

23. Stoll D., Rigoberta Menchu and the Story of all Poor Guatemalans, Boulder, Westview, 1999. 
dors. Ce gommage mettait en avant une autre dialectique de l'histoire où l'expérience du travail dans les plantations jouait un rôle central dans la socialisation. En effet, cette expérience du salariat avait pour corollaire une prise de conscience presque naturelle des rouages de l'exploitation capitaliste par des Indiens voués à la prolétarisation. Rien n'était plus intéressant aussi que cet escamotage de l'entrée en relation de son père avec la guérilla et le CUC, pour des motifs très pratiques. Toute une dynamique de conflits locaux entre coqs de villages, parfois articulés à des conflits socio-politiques régionaux ou nationaux - mais pas toujours - disparaissait purement et simplement pour laisser place à un schéma où l'exploitation et la répression entraînaient, immanquablement, une prise de conscience révolutionnaire, avait pour suite logique l'organisation des exploités et, ce faisant, leur rencontre avec les organisations révolutionnaires. Enfin, cette mise en récit éludait tout le jeu d'échanges de coups et de montée aux extrêmes qui avaient précipité le Guatemala dans la guerre interne au profit d'un récit qui avait considéré le développement de cette guerre comme le résultat inévitable des luttes de libération, par essence incompatibles avec un ordre oligarchique irréformable.

L'enquête menée par David Stoll avait ainsi permis de confronter un récit avec les faits, et ce faisant pas seulement de rétablir, dans la meilleure tradition de l'histoire positiviste, la vérité de situations mouvantes et parfois plus complexes qu'il n'y paraissait, mais de saisir dans toute sa subtilité la façon dont s'était construite la vision du monde d'une jeune femme quiché liée à la guérilla. Quels événements de sa trajectoire personnelle avaient été tenus pour capitaux par elle-même et par les gens qui l'avaient assistée lorsqu'elle avait construit son autobiographie ? Quels étaient ceux qui avaient été au contraire jugés accessoires ? Comment avait été construit son récit ? Les événements avaient-ils été enchainés les uns aux autres ? Comment avait-elle narré certains retournements de conjonctures ? Telles étaient les questions fondamentales auxquelles le travail très minutieux de Stoll donnait accès. Et je compris très vite que j'étais confronté à des questions du même type. Certains des récits publiés au début de la guerre, notamment celui d'Armstrong Wiggins 24, comme certains matériaux que j'avais pu recueillir à la même époque dans les camps de réfugiés, participaient du même type de rabotage des aspérités du contexte de l'époque. A les en croire, les communautés miskitues étaient toutes peu ou prou unies et opposées en bloc aux sandinistes, et ce dès le début des années 1980. Et une dialectique mettant au premier plan l'enchevêtrement du racisme et de la répression expliquait de façon parfaitement linéaire et cohérente l'enchaînement des événements. Les mobilisations en faveur des droits à la terre ou de la prise en compte et du respect des cultures miskitue et mayangna étaient des réactions «naturelles » face au racisme et la répression de ces « justes revendications » avait, là encore " naturellement », acculé les

24. Dirigeant miskitu membre du groupe fondateur de MISURASATA, qui s'exila aux EtatsUnis en 1981 où il devint l'un des membres de l'ONG amérindienne, Indian Law Ressource Center. 
jeunes activistes à la lutte armée. Les va-et-vient de certains acteurs, les conflits internes et les rivalités propres à la mouvance indianiste disparaissaient, et il en allait de même avec certains appuis extérieurs aux mobilisations. De même, les détails des premiers affrontements avec les sandinistes à Raïti et à San Carlos, affrontements qui avaient précédé le massacre de Leimus ou près de soixante-dix miskitus avaient péri assassinés, tandis que leurs femmes étaient violées, étaient souvent occultés au profit de récits où ces viols étaient à peine mentionnés. Aussi, à examiner mes premiers récits de vie, je pris conscience qu'il me faudrait établir de la façon la plus minutieuse les faits portant non seulement sur le déroulement des premiers affrontements avec les sandinistes - celui des manifestations et des premières échauffourées puis des combats quasi réguliers - mais plus encore sur certaines exactions commises durant la guerre ou lors de ses lendemains. Mettant à profit les enseignements de Paul Friedrich dans son étude des caciques révolutionnaires mexicains du Michoacan ${ }^{25}$, je devins expert dans l'art de croiser aussi bien les ragots ramassés aux hasards des conversations, parfois au contact de personnages fort peu reluisants, que les faits établis par des récits plus structurés et émanant a priori de personnes infiniment plus dignes d'estime et de respect. Fort de la connaissance de ces ragots qui suffisaient à mettre en cause des récits très structurés, j'en vins à oser formuler certaines questions, parfois assez indélicates, mais essentielles à la compréhension des processus que je voulais étudier et non moins essentiels à la compréhension des récits qui m'en étaient faits. Je ne m'en tins plus seulement aux consignes de David Brumble, qui avait très justement invité les analystes ou les co-auteurs «d'autobiographie modèle » à révéler la manière dont avaient été élaborés puis, au besoin, retravaillés, les dires des narrateurs. Je m'attachai aussi à saisir les écarts entre les narrations et les faits et à m'interroger sur le sens de ces écarts en m'en interdisant a priori toute interprétation essentialiste, culturelle ou politique. En effet, là encore, les polémiques qui avaient accompagné le travail de Stoll étaient particulièrement riches d'enseignements. Si celui-ci s'était parfois enfermé dans un rôle de dénonciateur des incohérences du récit de Rigoberta Menchu et de son instrumentalisation politique, les défenseurs de cette dernière avaient au contraire mis en avant le caractère «prophétique » de sa parole, $\mathrm{y}$ voyant une tradition $\mathrm{du}$ récit maya pour mieux en justifier les méandres ${ }^{26}$. A lire ces polémiques au regard des impératifs de ma propre enquête, je compris que si le travail de détective que l'on reprochait parfois à Stoll était, que cela plaise ou non, indispensable, il devait néanmoins s'articuler à une réflexion sur le sens de ces écarts entre les narrations et les faits, tout comme sur les visées manifestes et latentes de telles reconstructions. Comment Mono et Wari mettaient-ils en récit le surgissement de revendications indianistes, les premiers heurts avec les

25. Friedrich P., The Princess of Naranja. An Essay in Anthrobistorical Method, Austin, University of Texas Press, 1986.

26. Morales R. (ed.), Stoll-Menchu, la invencion de la memoria, Consucultura, Guatemala, 2001; Lempérière A., " Moi, Rigoberta Menchu. Témoignage d'une Indienne internationale ", Communications, n71, 2001, pp. 395-434 ; Le Bot Y., Rousseau C., «Rigoberta Menchu : naissance d'un sujet », Journal de la société des américanistes, vol.85, 1999, pp. 415-424. 
sandinistes ? Sur quoi insistaient-ils, que minoraient-ils, quels sens donner à leurs façons de relater les événements ? Comment leurs récits non seulement se recoupaient d'un point de vue factuel, mais comment chantaient-ils les mêmes événements à l'unisson, sur un même registre, ou au contraire de façon discordante ? Comment leurs récits s'articulaient-ils à d'autres autobiographies modèles où à la chronique rédigée par Santiago, toujours restée à l'état manuscrit et n'ayant connu aucune circulation en tant que texte?

Cette nécessité de croiser les faits et les relations de ceux-ci ne m’apparut pas moins nécessaire pour tout ce qui touchait au registre de la magie et de la sorcellerie, comme à celui du miracle et du merveilleux pendant la guerre. Peu ou jamais mentionnés par les analystes des affrontements Miskitus/sandinistes ${ }^{27}$, ces phénomènes, dont j'avais pu faire l'expérience directe lors de mes séjours dans la guérilla, n’en avaient pas moins joué un rôle à certains moments capital. J'avais ainsi pu voir comment certains présages influençaient de façon décisive la conduite des acteurs, comment certaines prières étaient récitées avec le plus grand sérieux avant de prendre la mer pour entrer clandestinement au Nicaragua, ou encore comment certains événements jugés bienheureux étaient interprétés comme autant de signes de l'élection divine ou de la faveur de puissances surnaturelles. Il me parut donc d'autant plus important de faire le point sur ces sujets de façon réfléchie qu'en apparence les conceptions en la matière semblaient à la fois souvent très floues et passablement contradictoires. Mes interlocuteurs me mentionnèrent, tantôt fort brièvement et en se gaussant, tantôt de la façon la plus détaillée et la plus empreinte de sérieux et de respect, le poids de certaines croyances et de certains rituels. On me raconta comment le mythe de la frégate chargée d'armes, qu'aurait promise sur son lit de mort la reine Victoria aux Miskitus, avait connu un regain de ferveur, tout comme la croyance en l'existence d'une liane rendant invisible. De même m'assura-t-on que Steadman Fagoth et certains de ses proches assesseurs, venus des rangs de la garde nationale, avaient plus d'une fois manifesté le souhait de faire appel à des sorciers du Belize ou de la Jamaïque pour faire mourir Tomas Borge et les autres dirigeants sandinistes. On me narra aussi la croyance de certains en l'efficacité d'amulettes protectrices, les tups, comme le danger représenté par les sirènes promptes à séduire et à s'emparer de combattants aguerris. On me vanta enfin la puissance de prières qui, à plus d'une occasion, avaient provoqué des pluies miraculeuses ayant constitué autant de protections de combattants obligés de cheminer à découvert, ou encore l'importance des rêves prémonitoires, qui là encore avaient évité bien des catastrophes.

Il me fallut apprendre à suivre les schémas logiques qui soutenaient ces raisonnements et à faire miennes les interprétations d'Edward Evans Pritchard 28 et de Paul Veyne ${ }^{29}$, c'est-à-dire à comprendre comment les gens

27. Ni les travaux de Bernard Nietschmann, ni ceux de Charles Hale n'en soufflent mot. 
croyaient cum grano salis, et comment la pensée magique et la foi pouvaient aller de pair avec un sens aigu de l'observation et de la déduction logique. Les mêmes qui m'offrirent de me mettre en contact avec un ancien capable de me fournir un bon tup me racontèrent en riant à gorge déployée comment ils avaient démasqué un charlatan vendeur de tups pare-balles en lui proposant d'essayer ceux-ci en tirant sur lui une rafale de kalachnikov. Ou encore comment ils s'étaient gaussé de Brooklyn Rivera qui avait un temps arboré un tup, lequel, du fait de son volume et de sa forme, ressemblait plus à un petit coussin qu'à un «vrai tup »!

J'appris aussi à repérer comment l'efficacité de la pensée magique ou la prégnance de certains schémas prophétiques ne tenait pas seulement au respect de la lettre de certaines injonctions, mais bien plus à un certain travail de la pensée et à la capacité des Miskitus de réélaborer en permanence à partir de ces structures d'opposition. La croyance dans la liane qui rendait invisible s'inscrivait dans une réactivation de croyance assez simple à déceler, puis à rapporter à la légende de Wis Wis. Cette légende racontait comment les habitants du village de Wiswis étaient en butte aux exactions d'un roitelet qui exigeait d'eux un tribut trop important. Et toutes leurs tentatives pour échapper à cette redevance faisaient qu'ils étaient en retour cruellement punis, en l'occurrence fouettés, sur ordre de ce roi tyrannique. Un jour qu'ils étaient partis à la chasse et qu'ils attachaient les pattes de pécaris qu'ils venaient de tuer avec une liane, ils découvrirent que le contact de celle-ci sur l'animal le rendait invisible. Ils imaginèrent aussitôt de l'utiliser pour se rendre invisibles eux aussi, et échapper ainsi aux persécutions. Et de fait, plusieurs anciens guérilleros me racontèrent comment certains de leurs premiers compagnons d'armes partirent à la recherche de cette liane. On me présenta même l'un d'entre eux, que l'on ne connaissait plus que sous le nom de cette liane (dar) tant il était souvent et longtemps parti à sa recherche. Là encore, les choses ne laissaient pas d'être ambiguës. Certains avaient très manifestement cru à la nécessité de chercher cette liane, afin de pouvoir prendre sans coup férir des postes militaires sandinistes dont ils ne pouvaient s'approcher sans être aisément détectés par des sentinelles observant depuis des positions fortifiées, d'où on pouvait les abattre à tout coup sans s'exposer. D'autres, et parfois les mêmes, avaient justifié des absences parfaitement irrégulières en prétextant être partis à la recherche de cette fameuse liane.

Les remises en circulation du mythe de la frégate prenaient parfois des détours d'une extrême subtilité. Certains avaient simplement opéré un déplacement : les Américains étaient venus remplacer les Britanniques comme fournisseurs d'une aide extérieure pour se libérer des Espagnols. D'autres avaient eu recours à des réaménagements plus complexes. On me raconta sur le mode

28. Evans Pritchard E., Wichtcraft, Oracles and Magic among the Azande, Londres, Oxford University Press, 1951.

29. Veyne P., Les Grecs ont-ils cru à leurs mythes ?, Paris, Le Seuil, 1983. 
goguenard comment une poignée des premiers guérilleros miskitus, les Astros et les Cruces, qui devaient d'ailleurs se heurter à Steadman Fagoth et engager les combats du «Noël rouge » (décembre 1981) 30 contre les sandinistes, avaient eu recours à des prières qui en appelaient à l'aide des extraterrestres et des astronautes pour que ceux-ci envoient des soucoupes volantes afin de les aider dans leurs combats contre les sandinistes. En apparence la chose portait à sourire et j'y vis tout d'abord, comme certains commentateurs Miskitus, des sortes de faux miracles et d'inventions dignes de certaines émissions radiophoniques à succès, ou de la littérature de science-fiction la plus bas de gamme. A $\mathrm{y}$ regarder de plus près, il y avait là une reprise très scrupuleuse des schèmes sous-tendant la prophétie de la reine Victoria. Les Miskitus, dans leur lutte contre ces substituts des Espagnols qu'étaient les sandinistes, en appelaient à un substitut des Anglais, les astronautes. Le déplacement tenait compte du rétrécissement du monde propre à la fin du vingtième siècle. La distance abyssale entre la Grande-Bretagne et la Moskitia, qui avait été celle du XIXe siècle était maintenue grâce au remplacement des Britanniques par les extraterrestres ; le vecteur du contact restait un vaisseau, non plus maritime mais spatial. Et c'était donc en bonne logique qu'une soucoupe volante se substituait à une frégate. Et c'était toujours en bonne logique que mon ami Mono et d'autres moraves se gaussaient de cette prédiction qui leur apparaissait comme le type même de la fausse prophétie, même s'ils avaient pu en mesurer l'efficacité. En effet, les premiers moraves n'avaient eu de cesse que de combattre le mythe de la frégate ${ }^{31}$. Ces premiers combattants avaient d'ailleurs aussi opté pour une auto-dénomination, Astros, qui là encore faisait résonner deux thèmes au cœur de la tradition miskitue : la reprise modernisante de la prophétie de la frégate, mais aussi l'histoire d'Aimapaya, ce couple d'enfants, frère et sœur, qui étant les seuls à avoir survécu à un déluge au cours duquel avaient péri tous les autres habitants, avaient engendré les Miskitus avant de devenir au lendemain de leur mort deux étoiles dont, selon la formule de la fin du conte, «le brillant éclaire pour toujours » les Miskitus ${ }^{32}$. Là encore, l'allusion était limpide, les Astros étaient destinés à refonder les miskitus menacés par la guerre que leurs menaient les sandinistes. Il était aussi un autre épisode miraculeux de l'histoire des Astros, là toujours raconté avec la plus grande ferveur. Au lendemain de leur sécession d'avec Fagoth, la petite troupe de guérilleros, après avoir cheminé toute la journée, s'était installée dans la cabane d'un cultivateur à côté d'une rizière et d'une petite bananeraie. Ils y avaient trouvé une marmite et quelques provisions. Reina Jack, la seule recrue féminine de la troupe, et Tigre, avaient alors commencé à cuisiner le peu de nourriture présente. Et là plus, ils servaient à manger à leurs compagnons, plus la petite marmite sem-

30. Les sandinistes baptisèrent ainsi les combats engagés en décembre 1981 par les guérilleros miskitus à Raiti et à San Carlos, deux bourgs du Rio Coco.

31. On trouvera dans les mémoires de l'évêque miskitu Karl Mueller une très nette condamnation de cette croyance : Mueller K., Among Creoles, Miskitos and Sumos. Eastern Nicaragua and its Moravian Missions, Bethlehem, The Comenius Press, 1932.

32 . J'utilise ici la version de recueillie par Ana Rosa Fagoth, publiée par ses soins dans la réédition des bulletins Tininiska en deux volumes : Tininiska tome 1, 2003, pp. 19-27. 
blait remplie ! Mieux : tous purent se servir jusqu'à satiété. Et quand Tigre me raconta le premier ce miracle en présence de Mono, le recueillement avec lequel il évoqua cet épisode m'invita immédiatement à y lire la double reprise de thématiques au centre de la religion morave : la parabole de la multiplication des pains, et l'invention de la Cène de Herrnhut. On se souvient que lors de la fondation de la première communauté morave sur ses domaines, Zinzendorf avait marqué la réconciliation entre les Saxons et les Bohémiens en organisant une agape. Ici, à nouveau une scission est sanctionnée et sanctifiée de la même manière, et la justesse de la décision des Astros est soulignée par le miracle de la multiplication de la nourriture.

J'appris donc à suivre comment se construisaient les attestations sociales des miracles ou du merveilleux, et ce faisant à en donner les descriptions les plus exactes. Et je pus dès lors commencer à jauger l'importance sociale de ces phénomènes en ayant toujours présent à l'esprit que le «faux » coexistait inévitablement avec le «vrai », et que la seule manière de les départager était une fois de plus le dialogue le plus ouvert possible.

\section{Des positions fluctuantes : témoins et enquêteur}

Cette capacité nouvelle à repérer des différences parfois fondamentales dans les registres d'expression de mes multiples interlocuteurs me conduisit moins à tenter de figer ces différences dans une typologie de mes témoinsinformateurs qu'à m'interroger sur les processus qui les sous-tendaient. Ces différences n'étaient en effet nullement des données stables. Elles s'inscrivaient dans un jeu mouvant d'interactions entre des pôles eux-mêmes changeants. Je fus tantôt dans un échange entre deux pôles (moi et le ou les témoins) tantôt dans des échanges triangulaires où les personnes avec lesquelles je m'entretenais me furent présentées par des tiers qui formèrent un véritable pont entre nous. Enfin, si certaines de mes rencontres s'inscrivirent dans des rapports relativement stables, d'autres furent au contraire marquées par de constantes évolutions.

Construites moins sur la volonté de dialoguer que sur celle de témoigner pour la postérité, les relations avec des interlocuteurs comme Laurita et Tillith Mollins, Rogelio Bob ou Nicodemo Serapio me parurent marquées par le modèle de la déposition judiciaire ou de l'enquête ethnographique la plus traditionnelle. Je ne fus pour eux qu'une sorte de scribe chargé d'enregistrer leurs dépositions et de les archiver; un scribe d'ailleurs " autorisé » et qui leur avait été présenté par un médiateur de confiance, le plus souvent Samuel Kittlé ou Adam Artola. Sans eux, ou d'autres de mes anciennes connaissances de la guérilla, aucun entretien de ce type n'aurait été possible. Lui, Wari et d'autres de mes anciens élèves de El Atillo, non seulement me présentèrent leurs parents et les invitèrent (et parfois même les sommèrent, ce fut le cas de Rogelio Bob) 
de me parler sans ambages "pour qu'il reste quelque chose de tout cela»! Il y avait là pour Samuel et mes anciens étudiants comme des contre-dons en retour de mes cours dispensés dans le passé. Je leur avais enseigné l'histoire du vingtième siècle et d'un certain nombre de mouvements armés aux obédiences les plus variées ; ils avaient à cœur de me présenter l'histoire de la Moskitia au travers de celles de leurs familles. Comme en écho à nos échanges d'El Atillo où j'avais professé, leurs histoires et celles des leurs s'inscrivirent dans une manière d'égalité avec celles du Viêt-minh, des maquis algériens ou de ceux d'Amilcar Cabral, des guérillas latino-américaines sur lesquelles nous avions travaillé de concert. Et de fait transformèrent-ils leurs parents et leurs connaissances en autant de «cas » qui me furent soumis et présentés dans une sorte de symétrie avec les exemples qui avaient nourri mes cours et nos débats. Ces témoins invités à déposer devant moi furent pour eux autant d'équivalents des articles de revue (Esprit, Hérodote et Vuelta) et des auteurs, comme Raymond Aron et Gérard Chaliand, Marc Ferro et Octavio Paz, Yvon Le Bot et Gabriel Zaïd, que je leur avais présenté. Ils avaient pu appréhender un monde plus vaste que le leur, et réinscrire leurs expériences de la guérilla dans des processus plus larges en les comparant à d'autres; ils me donnèrent en retour la possibilité d'accéder dans tous leurs détails aux méandres de l'histoire des Miskitus. Du coup, le type d'échange que j'eus avec ces «témoins présentés » n'était pas destiné au départ à s'inscrire dans une relation marquée par un souci de conversation de longue durée, pas plus que dans une quelconque réflexivité partagée. Pour ces témoins, comme pour ceux qui d'une certaine façon les citaient à comparaître, il importait avant tout que les dires soient à la fois les plus clairs possible, soient recueillis minutieusement, et deviennent ainsi susceptibles d'être transmis à la postérité en devenant les pièces et les documents d'un futur livre. Une fois que ces témoins avaient fait leur déposition, l'affaire était pour eux, d'une certaine façon, close. Sans doute étaient-ils en attente de voir leurs dires publiés, et que je leur apporte un exemplaire de l'article ou du livre où apparaitraient leurs témoignages. Mais marqués par le précédent de Mary Helms, dont le livre sur Asang n'avait été publié en anglais que six années après son travail de terrain, et près de dix ans plus tard en espagnol, et qui de surcroît avait peu circulé, ils n'escomptaient nullement me voir immédiatement leur rendre visite avec un livre à leur montrer. La chose fut d'ailleurs parfaitement claire pour ceux qui nous avaient mis en contact. Quand ils me présentèrent à ces témoins, Mono, Wari ou d'autres reprirent souvent, presque mots pour mots, ce que j'avais pu leur dire sur le temps nécessaire à un travail de qualité, et sur ma volonté de ne publier que des choses dûment réfléchies.

Si ces premières rencontres se construisirent dans un contexte marqué par la stabilité de positions, mes relations et mes dialogues avec certaines de mes autres connaissances, tels Wari, Tigre ou Kenneth Serapio, s'inscrivirent d'emblée dans des processus marqués par de perpétuels remaniements. Le premier 
motif de ces oscillations fut pour une part fonction des zones d'ombres de certains témoignages. L'histoire de Tigre en comportait d'incontestables, dans lesquelles nous n'entrâmes pas, mais qui, je le perçus très vite, pourraient me réserver quelques surprises, notamment sur l'époque où il avait été membre des rangs subalternes de la Garde nationale, ou même sur celle des premiers maquis miskitus commandés par Nicodemo Serapio, à l'époque des Astros et des Cruces. La presse sandiniste avait alors évoqué des mutilations de cadavres et des exécutions cruelles. Etait-ce pure propagande ? Ces faits étaient-ils au contraire avérés ? Dans ce dernier cas, qui en étaient les auteurs, et qui les responsables? Je sus qu'avec le temps, la lumière pourrait bien se faire, soit pour confirmer ces accusations et révéler les visages des auteurs des crimes, soit pour les infirmer. De telles mises au point pourraient bien évidemment faire basculer mes relations avec le témoin, s'il s'avérait qu'il en était un des auteurs. Il me parut dès lors que le dialogue que nous avions commencé à nouer pourrait bien devenir impossible, car je prendrais la figure au mieux d'un procureur à charge, au pire d'un «traître » ayant joué de sa proximité avec son interlocuteur pour mieux le piéger ensuite. A l'inverse, la multiplication des rencontres pouvait déclencher une dynamique d'échanges intellectuels plus suivie, et ce d'autant plus que je serais à même de prouver que ces accusations n'étaient qu'affabulations. Je fus amené au même constat avec Kenneth Serapio. Ses offres de collaboration à la BICU, sa passion pour les discussions sur la situation politique et sociale du moment, pouvaient là aussi se révéler riches d'échanges intellectuels tout aussi fournis que ceux que j'avais déjà avec Mono ou Wari. Une autre possibilité n'était nullement à exclure : la transformation de Tigre ou de Kenneth en colporteurs de paroles tout aussi figées et convenues que celles des têtes de file du Conseil des anciens et de leurs conseillers. L'un et l'autre aspiraient à des responsabilités politiques. Le premier faisait partie des anciens guérilleros cherchant l'alliance avec les libéraux proches du nouveau président de la République, Arnoldo Aleman. Sans doute, quand je le rencontrai, ne décoléra-t-il pas contre l'homme de paille de Fagoth, Gerardo Tomas, un des anciens du séminaire de El Atillo devenu maire de Waspam. Mais n'était-il pas lui aussi potentiellement corruptible ? Kenneth, qui avait fondé un parti appelant très intelligemment à l'union de dissidents du sandinisme et des réformateurs de la guérilla, n'était-il pas lui aussi susceptible de revirements ? La question méritait d'autant plus d'être posée que le contexte favorisait grandement des manières d'arrangements prosaïques au jour le jour. En effet, les expériences répétées de promesses non tenues étant nombreuses, beaucoup en étaient venus à considérer que les arrangements au jour le jour étaient la seule solution raisonnable. Ainsi, lors des débuts de la révolution, comme lors des premiers moments de la guérilla, les dirigeants sandinistes, puis ceux de la Contra, avaient promis l'entrée à brève échéance dans de véritables pays de cocagnes. Peu avare de promesses bibliques, Fagoth avait promis un avenir où «couleraient le lait et le miel ». Bien évidemment, ces royaumes d'abondance n'étaient jamais advenus. Les 
gens avaient, au contraire, fait l'expérience de la plus grande détresse matérielle et de l'incertitude, et avaient été les témoins d'incessantes affaires de détournements de fonds durant la guerre, tant du côté des sandinistes que de celui des contras, puis lors de la présidence de Doña Violeta (Barrios de Chamorro, de 1990 à 1996). Tout cela avait poussé beaucoup de Miskitus à opter pour un certain cynisme. Aussi ne pouvais-je exclure qu'en cas de succès politique, Tigre et Kenneth Serapio ne se plient aux usages en vigueur, qui consistaient à multiplier les arrangements les plus scabreux et les détournements de fonds au profit des politiciens et de leurs familles. Et je savais parfaitement qu'il me serait dès lors assez difficile de converser avec eux avec la liberté et la franchise qui avaient caractérisé nos premiers entretiens. Sans jamais être face à des oscillations de la sorte, je commençai d'ailleurs très vite à expérimenter des variations dans mes relations avec Adam. Très enthousiaste au début, il se lassa de la lenteur de mon enquête comme de mes sempiternelles questions, et de ma manie de recouper inlassablement mes informations. Par ailleurs, de plus en plus pris par son rôle de procureur à Puerto Cabezas, amené à rencontrer les autorités politiques libérales et les représentants du président dans la région, il cessa de parler avec la même liberté avec moi craignant, non sans raison, que je donne écho à des informations qui pourraient nuire à sa carrière. Sans que rien ne soit dit, sans que notre proximité soit remise en cause, nos relations s'étiolèrent quelque peu, nous conversâmes moins, et chaque jour de choses plus anodines, tout en prenant soin de part et d'autre de ne jamais nous perdre vraiment de vue lors de mes visites à Puerto Cabezas.

A l'inverse, la relation que je renouai avec Mono s'inscrivit dans un processus de construction d'un compagnonnage intellectuel et moral fondé sur un intérêt mutuel pour un projet intellectuel, comme sur une estime réciproque qui me parut devoir aller crescendo au fil de nos échanges. Nul doute que notre complicité reposait à la base sur nos premiers échanges, lorsque je fis sa rencontre le $1^{\text {er }}$ mai 1984, puis lors du séminaire d'El Atillo. Ce $1^{\text {er }}$ mai miskitu avait été pour moi une manière de révélation. J'avais découvert au tout début de mon séjour dans la guérilla le spectacle peu attendu d'un commandant de troupe, Mono en l'occurrence, prenant à partie le commandant en chef de la guérilla devant quelque cent cinquante à deux cents guérilleros. Je l'avais entendu développer une critique minutieuse des erreurs tactiques et stratégiques de l'état-major, de ses propensions dictatoriales et prévaricatrices. Son franc parler comme son style tranquillement mesuré, et de la sorte d'autant plus caustique, son apparence de Hô Chi Minh amérindien, son indéniable courage, furent autant d'éléments qui me séduisirent et m'impressionnèrent. Son intelligence et sa curiosité intellectuelle, que je n'avais pu qu'entrevoir à ce moment mais que j'avais achevé de découvrir lors de mes cours, devaient finir de me persuader de la qualité du personnage et me conduire à multiplier les échanges avec lui. Comme Mono me l'avait raconté à plusieurs reprises par 
la suite, j'étais arrivé au bon moment, et les propos que j'avais pu tenir lors de mes cours comme lors de nos discussions à bâtons rompus avaient été d'une importance décisive pour lui et les autres réformateurs. J’avais formulé sans trop le savoir des propositions et des critiques qu'ils attendaient. Lui-même et d'autres avaient par ailleurs repris à leur compte telle ou telle de mes analyses pour prendre contact avec les commandants des troupes de guérilleros inféodés à Brooklyn Rivera et faire le point avec eux sur la situation politique et militaire, puis se lancer dans l'aventure de la Reforma. L'absence de zone d'ombre dans la vie de Mono et son intégrité foncière facilitaient nos échanges intellectuels. Je savais que je n'avais nulle mauvaise surprise à redouter, comme j'en eus rapidement la preuve. En effet, alors que certains de mes autres interlocuteurs faisaient l'objet de commérages, parfois très peu flatteurs, différents quidams de rencontre me reprochèrent d'interroger des «corrompus ", des "profiteurs ", des « coureurs » ou des « ivrognes ", mais jamais Samuel n'encourut l'ombre de ces critiques. De même, si on me fit parfois comprendre que certains avaient soit participé à des exécutions sommaires au sein de la guérilla, soit mené des vendettas personnelles, les seuls reproches, que je pus entendre à son encontre furent d'une toute autre nature. Ils visaient son intransigeance face aux vols, aux manquements à la discipline à l'intérieur de la guérilla. On me raconta ainsi comment, au lendemain d'un coup de force manqué de Steadman Fagoth, il n'avait pas hésité à puiser des arguments dans l'Ancien Testament pour exiger à son encontre un châtiment exemplaire. Quelles qu'aient été mes réserves face à l'application de la peine de mort pour trahison, je n'étais nullement face à une série de débordements dont il me serait difficile de parler avec Mono, mais au contraire devant une série de faits que nous pourrions évoquer sans faux semblants et sans qu'il ait l'impression que je me transforme en procureur faisant fi des liens amicaux. De même pouvait-il être sans crainte par rapport à mon travail de publiciste. S'il avait su très tôt que je ne dissimulerai pas des événements qui pouvaient donner lieu à des critiques, au besoin dommageables pour sa cause et son image, il savait en même temps que je n'étais à la recherche ni de "sensationnel », ni de «scandales ", mais que je voulais comprendre le sens de leurs actions, que j'avais le souci d'étayer mes explications et d'établir les faits que j'avançais. Il savait aussi que, si besoin était, je respecterais l'anonymat de mes sources. Si, fort de ces premiers échanges comme de cette intégrité de Mono, j'escomptai que la qualité des relations que nous tissions ne pourrait aller que se solidifiant dans un dialogue interminable sur l'histoire de la Moskitia, il me parut qu'il faisait lui aussi un pari assez semblable sur l'avenir. Nos échanges nous avaient appris à l'un comme à l'autre, à voir de manière décentrée par rapport à nos perspectives habituelles; nous y avions gagné l'un et l'autre en hauteur et en largeur de vues. J'accédais par son entremise à la culture miskitue dans toutes ses subtilités. J'entrevoyais une aventure familiale déployée sur plusieurs générations, où d'anciens guerriers semi-nomades s'étaient sédentarisés et convertis au protestantisme. Je découvrais toutes les stratégies d'une fratrie en vue d'améliorer ses conditions de vie et de promouvoir le développement 
d'une région longtemps en marge des grands courants d'échanges. Mono déployait devant moi à la fois des traditions amérindiennes, et toutes les traditions orales qui s'y associaient, mais aussi les extraordinaires capacités du métissage actif développées par les Miskitus depuis le seizième siècle. Il était enfin un exemple particulièrement frappant des aptitudes des Miskitus à tout un travail réflexif qui plongeait ses racines dans le piétisme. Il accédait à travers moi aux habitus intellectuels français et plus particulièrement à ceux d'une famille surplombée par la figure d'un grand-père humaniste érasmien. Il touchait au travers de nos conversations à toute une tradition critique occidentale nourrie d'un mélange de rationalisme laïque et d'un ethos démocratique et égalitaire. Il découvrait les ressorts d'une sociabilité fondée sur des codes égalitaires qui, chose parfaitement étrange au regard de la nouveauté de l'égalité démocratique en Amérique latine, plongeait dans une tradition ancrée dans le dix-neuvième siècle. De même que j'enrichis mes capacités réflexives à entendre sa manière de présenter son histoire, celle de sa famille ou de sa région et de leur guerre contre les sandinistes, j'en vins à voir d'un autre oil mes proches ou certains codes, en apparence les mieux établis, de mon monde social. De son côté, il en vint à questionner certains habitus nicaraguayens ou miskitus.

\section{Conclusion : établir les faits, les interpréter et les mettre en sens}

Si l'accumulation des pièces permettant de composer une mosaïque de la Moskitia, certains détours par l'histoire centraméricaine et l'analyse d'expériences parallèles à celle des Miskitus m'ont permis de lever un certain nombre d'équivoques liées aux conditions de mes premières enquêtes en 1982, puis en 1984, certaines tensions se sont maintenues, et ce, sans doute de façon féconde pour le travail intellectuel. Nul doute de ce point de vue que mes connaissances accumulées tout au long de ces enquêtes et celles tirées de travaux scientifiques menés parallèlement aux miens permettent aujourd'hui d'examiner chacune des pièces que j'entends intégrer à ma mosaïque de la Moskitia avec un œil beaucoup plus averti qu'il y a une vingtaine d'années. Certains miracles ou certaines pratiques magiques, les imbrications du religieux et $\mathrm{du}$ politique, l'incorporation d'babitus moraves par les Miskitus, ne sont plus des éléments discrets difficiles à appréhender, comme à mes débuts. Beaucoup prennent place dans des séries d'événements identiques et sont donc susceptibles d'analyses beaucoup plus riches. Ils sont à la fois comparables avec d'autres survenus en Moskitia ou dans le restant du Nicaragua. Les jeux de pouvoir et les démonstrations de force, comme la pratique des discussions collectives que je pus décrire au sortir de la guérilla, sans pouvoir en restituer le contexte ni en mesurer complètement la portée quand j'écrivis un article pour la revue anarchiste italienne Volonta ${ }^{33}$, sont devenues passibles d'analyses en rapport avec l'histoire de la guérilla elle-même, ou bien encore avec les contextes nicaraguayen et centraméricain. 
Fort de mes nouvelles connaissances, je pus ainsi décrire le surgissement des contre-pouvoirs des comandantes au sein de la guérilla ${ }^{34}$, puis la montée en force de ceux-ci à l'encontre des premiers dirigeants de MISURASATA ${ }^{35}$. Je fus enfin capable de resituer la geste des guérilleros miskitus dans la série qu'ils formaient avec d'autres acteurs armés dans le jeu politique nicaraguayen tout au long du XIX et du XXe siècle; et grâce à ce travail de comparaison d'en mettre à jour des significations jusqu'alors peu visibles ${ }^{36}$. Ma meilleure connaissance du piétisme morave me servit à faire le lien entre certaines pratiques de la discussion collective chez les Miskitus et au sein de la guérilla avec l'incorporation des habitus piétistes. La familiarité acquise avec la religion morave m'en rendit intelligibles certaines dimensions qui incontestablement me facilitèrent la tâche. Enfin, mes séjours répétés sur le Rio Coco, comme mes discussions avec certains responsables d'ONG, m'ouvrirent les yeux sur les impasses des projets de développement en Moskitia, tout comme sur les effets pervers de l'aide internationale distribuée au lendemain de l'ouragan Mitch ${ }^{37}$.

Ce processus d'accumulation des connaissances, qui alla de pair avec un éloignement temporel croissant des événements les plus sujets à polémiques, tel le Noël rouge et la tuerie de Leimus (décembre 1981) ${ }^{38}$, n’a pas pour autant été coextensif de l'installation des observateurs de la Moskitia dans une position de surplomb et d'extériorité face aux événements et aux acteurs. Les interactions entre les processus de connaissance et d'objectivation et les jeux socio-politiques restent d'actualité. Si le champ des idéologies a été remanié, si l'heure n'est plus ni aux combats anti-totalitaires, ni au refus de la remise en selle des pratiques autoritaires, la volonté d'établir le plus rigoureusement les faits, de distinguer le vrai du faux sans se préoccuper des conséquences politiques d'un tel travail critique, continue de susciter des réticences. Ces réticences, faut-il le préciser, ne sont nullement l'apanage des protagonistes : elles restent à l'honneur chez certains chercheurs. Ainsi, les questions relatives au nombre de mineurs tués en décembre 1981 par les militaires sandinistes à Leimus et dans les environs, aux responsabilités du haut commandement sandiniste en la matière, d'autres sur la manière dont furent assassinés les prisonniers faits par les Astros et les Cruces lors des attaques de San Carlos avant

33 . Bataillon G., « Moskitia 1984 », op. cit.

34. Bataillon G., «Comandantes, état-major et guérilleros : jeux de pouvoirs à l'intérieur de la guérilla miskitu (Nicaragua, 1981-1984)», op. cit.

35 . Bataillon G., " Guerre et assemblée, découverte et apprentissage de la démocratie chez les Miskitus nicaraguayens (1981-1988)», op. cit.

36. Bataillon G., «De Sandino aux contras. Formes et pratiques de la guerre au Nicaragua », op. cit.

37. Bataillon G., "Cambios culturales y sociopoliticos en las comunidades Mayangnas y Miskitus del RioBocay y el alto Rio Coco ", op. cit. ; "Wangki/Rio Coco : de l'après-guerre aux catastrophes naturelles ", op. cit.

38. Massacre commis par l'armée à Leimus les 23 et 24 décembre 1981 à l'encontre de 110 hommes miskitus en représailles des attaques quelques jours plus tôt des postes militaires de San Carlos et de Raïti, dénommées attaques du Noël rouge par les sandinistes. 
d'être remis aux responsables de bases de la guérilla dont le chef était Steadman Fagoth, restent d'une actualité brûlante. Les exécutants de ces tueries et des viols qui les accompagnèrent, les responsables politico-militaires qui ordonnèrent ou couvrirent ces crimes, font toujours partie du personnel politique nicaraguayen, parfois au plus haut niveau. Ecrire sur ces sujets diminue ou accroît les ressources des protagonistes politiques et sociaux dans leurs compétitions pour le pouvoir. On ne saurait ignorer leurs effets de légitimation comme de stigmatisation. Des travaux ne relevant pas d'une anthropologie historique des massacres ${ }^{39}$, mais portant sur la question du pouvoir au sein des guérillas comme sur la question du développement, pèsent eux aussi sur l'actualité la plus immédiate. Bon nombre des acteurs militaires dont j'ai analysé les gestes et les stratégies sont devenus des acteurs politiques. Les responsables de l'échec de certaines opérations de développement sur le Rio Coco, comme du piratage de certaines enquêtes sur la tenure foncière, ou du détournement de fonds destinés à la reconstruction au lendemain de l'ouragan Mitch, sont des personnages publics. Certains sont parfois célébrés pour leur apport à la cause des minorités ethniques ou à celle du droit des femmes indigènes. Les articles scientifiques entrent donc en résonance avec les enjeux socio-politiques et les débats du présent. Les remarques formulées par Pierre VidalNaquet dans les années 1980 sur la nécessité pour les sciences sociales de se confronter aux faits qui sont au cœur des passions politiques ("qu'une idéologie s'empare d'un fait ne supprime pas l'existence de celui-ci... ») sont toujours d'une parfaite actualité, un quart de siècle plus tard. Sa méthode aussi : établir les faits pour s'approcher au plus près des réalités qui existent en tant que " choses en soi », même si elles sont reconstruites par les témoins. Cette nécessité d'une anthropologie, d'une histoire ou d'une sociologie, toutes trois soucieuses de distinguer rigoureusement entre le vrai et le faux, reste aussi impérieuse que par le passé. Elle doit conduire à établir les faits, à les présenter et à en restituer le contexte ${ }^{40}$. Une telle démarche permet d'éviter l'obsession mémorielle lourde, selon les mots de Michelet, de «tendresses oublieuses » et de «larmes vites séchées " 41 .

Comme Claude Lefort l'a bien écrit, les traditions anthropologique, historique ou sociologique ont partie liée avec l'histoire du cheminement démocratique; l'expérience démocratique suppose une opération de désincorporation des sphères du pouvoir et du savoir. En régime démocratique, le pouvoir obéit à des règles de remise en cause périodique et demeure un « lieu vide», inappropriable définitivement par quiconque, individu, groupe social, reli-

39. Je reprends cette formule de Dewerpe A., Charonne 8 février 1962. Anthropologie historique d'un massacre d'Etat, Paris, Gallimard, 2006.

40. Je suis sur ce point les remarques formulées par Carlos Ginzburg dans le sillage de Pierre Vidal-Naquet: Ginzburg C., Un seul témoin, Paris, Bayard, 2007, p. 66.

41 . Je tire ces propos de Michelet, voir le recueil de textes de cet historien rassemblé par Claude Lefort : Michelet J., La Cité des vivants et des morts, textes rassemblés par C. Lefort, Paris, Belin, 2002, voir surtout pp. 64-65. 
gieux ou politique ; les conflits y sont reconnus et font l'objet d'une symbolisation. Le registre de la connaissance a partie liée avec l'expérience démocratique et il se déploie selon des principes qui entrent en résonance avec ceux qui régissent la sphère $\mathrm{du}$ pouvoir. $\mathrm{La}$ recherche $\mathrm{du}$ bien et $\mathrm{du}$ vrai suppose l'épreuve de la confrontation et de la discussion, comme celle du doute méthodique pour arriver à « faire le point ». L'idée d'un grand juge non questionnable, installé dans une position de détenteur de la vérité, apparâit non seulement comme frappée d'illégitimité, mais plus encore comme une perversion de l'activité de la pensée. L'opération de connaissance suppose que l'interprète des faits et des événements, qui forment la trame de l'histoire, ait pour visée première le dévoilement de la verita effetuale. S’il passe par les dires des témoins, il ne saurait jamais pour autant les instituer en autorités indiscutables. Leurs dires doivent être pris en considération, c'est-à-dire passés au crible de la critique et au trébuchet de la distinction entre le vrai et le faux. Enfin, cette absence de toute idée de l'existence d'un garant ultime au fondement de l'ordre social, qui est le propre de l'expérience démocratique, s'accompagne d'une valorisation de l'activité réflexive comme indispensable au bon fonctionnement de cette même expérience. Tout mon commerce avec les Miskitus se déploya et reste marqué par cette dimension critique. Elle permit que soient acceptées mes enquêtes sur la guerre, puis sur l'histoire et la culture des Miskitus et des autres habitants de la Moskitia. Elle est ce qui me permet de poursuivre mon travail de réflexion, non seulement sur l'histoire proche ou lointaine, mais aussi sur le présent. 